\title{
Improved vacuum system for high-power proton beam operation of the rapid cycling synchrotron
}

\author{
Junichiro Kamiya $\odot,{ }^{*}$ Hirofumi Kotoku, Syunta Kurosawa, Kazuhiro Takano, \\ Toru Yanagibashi, and Kazami Yamamoto \\ Japan Atomic Energy Agency, 2-4 Shirakata, Tokai, Naka, Ibaraki 319-1195, Japan \\ Kaoru Wada \\ Tokyo Electronics Co. Ltd. 2-22-7 Honcho, Kokubunji, Tokyo 185-0012, Japan
}

(Received 4 August 2020; accepted 19 July 2021; published 19 August 2021)

\begin{abstract}
The vacuum system in the rapid-cycling synchrotron of the Japan Proton Accelerator Research Complex has been operated for more than 10 years. It becomes evident that the high-power beam operation has more powerful effects on the vacuum system than expected at the time of the design. Those effects of the high-power beam are categorized into two types of events, the malfunction of vacuum equipment and the large pressure rise. A specific example of the former event is the failure of the turbomolecular pump (TMP) controller by the full loss of a single-shot high-intensity beam. The TMP itself is also damaged by a bearing crush due to a touch down without braking the rotor. We have developed a TMP controller that can connect with long power and control cables of more than $200 \mathrm{~m}$ lengths. This length makes the controller be installed in a control room where there is no radiation influence. The TMP with high-strength bearing has been also developed. The TMP system becomes tolerant to a large loss of the high-intensity beam by using such a controller and a TMP. The latter event is an extreme pressure rise with increasing the beam power. The dynamic pressure behaviors during the beam operations are investigated to understand the outgassing mechanism by the beam. It is indicated that the pressure rise mechanism is a result of the ion-stimulated gas desorption. The analytical calculation based on the ion-stimulated gas desorption model well reproduces the measured dynamic pressure. The calculation also shows that, among the parameters, a larger pumping speed per unit length $s$ and a smaller initial surface density of the adsorbed molecules $q_{0}$ are required to suppress the extreme pressure rise. The nonevaporable getter (NEG) pumps are additionally installed to obtain the larger $s$ and smaller $q_{0}$. It is confirmed by simulating the beam line pressure distribution that the additional NEG pumps are effective to obtain the larger $s$ in every position along the beam line. The ability of the NEG pump to keep a low pressure during the vacuum system shutdown, which can contribute to maintain the small $q_{0}$, is examined by the buildup test. It is finally confirmed that the dynamic pressure during the high-power beam is effectually suppressed.
\end{abstract}

DOI: 10.1103/PhysRevAccelBeams.24.083201

\section{INTRODUCTION}

\section{A. Overview of the rapid-cycling synchrotron vacuum system}

The $3 \mathrm{GeV}$ rapid-cycling synchrotron (RCS) of the Japan Proton Accelerator Research Complex (J-PARC) has provided the beam to users for more than 10 years since the first success of the acceleration and extraction of the $3 \mathrm{GeV}$

\footnotetext{
junichiro.kamiya@j-parc.jp
}

Published by the American Physical Society under the terms of the Creative Commons Attribution 4.0 International license. Further distribution of this work must maintain attribution to the author(s) and the published article's title, journal citation, and DOI. proton [1]. The RCS delivers $3 \mathrm{GeV}$ proton beams to the materials and life science experimental facility (MLF) and the main ring synchrotron. The main ring synchrotron delivers $30 \mathrm{GeV}$ proton beams to the Neutrino Experimental Facility and the Hadron Experimental Facility [2]. Table I summarizes the RCS design parameters [3]. The RCS accelerates the $400 \mathrm{MeV}$ protons injected from the linac up to $3 \mathrm{GeV}$ with a repetition rate of $25 \mathrm{~Hz}$. The ramping pattern is sinusoidal. Thus, the acceleration time from the beam injection to the extraction is $20 \mathrm{~ms}$. The design output beam power of the RCS is $1 \mathrm{MW}$, which is achieved by extracting the $8.3 \times 10^{13}$ protons per pulse of $3 \mathrm{GeV}$ energy at $25 \mathrm{~Hz}$. The RCS has increased the beam power for the user operation toward the design value of $1 \mathrm{MW}$ step by step. As of 2020, the RCS has experienced simultaneous beam operations with the output beam power of $500 \mathrm{~kW}$ to the 
TABLE I. Design parameters of the J-PARC RCS [3].

\begin{tabular}{lc}
\hline \hline Circumferences & $348.3 \mathrm{~m}$ \\
Injection energy & $400 \mathrm{MeV}$ \\
Extraction energy & $3 \mathrm{GeV}$ \\
Repetition rate & $25 \mathrm{~Hz}$ \\
Acceleration time & $20 \mathrm{~ms}$ \\
Ramping pattern & Sinusoidal \\
Number of particle per pulse & $8.33 \times 10^{13}$ \\
Output beam power & $1 \mathrm{MW}$ \\
\hline \hline
\end{tabular}

MLF and $510 \mathrm{~kW}$ to the Neutrino Experimental Facility. Also, the RCS has experienced simultaneous operations with $600 \mathrm{~kW}$ beam power to the MLF and $50 \mathrm{~kW}$ to the Hadron Experimental Facility. Furthermore, the RCS has tested the $1 \mathrm{MW}$ beam operation three times for $1 \mathrm{~h}$ in 2018 , $10.5 \mathrm{~h}$ in 2019, and 1.5 days in 2020.

The RCS vacuum system has been constructed according to the following design concepts [4]: (1) vacuum components in the beam line, such as beam pipes and bellows, should have sufficiently large aperture sizes compared with the cross section of the beam, (2) the beam line components are required to have a low-residual radioactivation characteristic and the components in the accelerator tunnel should be resistant to radiation, (3) eddy currents in the beam pipes caused by the rapidly changing magnetic field should be avoided, and (4) outgassing of the vacuum components should be minimized and enough pumping speed should be ensured over a wide range of pressures to evacuate the unexpected additional outgassing caused by the beam.

Figure 1 shows the layout of the RCS vacuum system $[4,5]$. The RCS consists of three straight sections and three arc sections - injection, extraction, RF, first arc, second arc, and third arc sections. Metal gate valves are installed between each section. The typical beam pipe diameter is about $250 \mathrm{~mm}$. For the beam injection area, the diameter is $400-500 \mathrm{~mm}$ to accept both the circulating and the injecting beam. The beam pipe diameter of the beam extraction area is a similar size to that of the injection area to accept both the circulating and extracting beam. Titanium is used for the beam pipes and bellows material due to the low-residual radiation characteristics [6]. The beam pipes in the magnets are made of alumina ceramics to prevent the eddy current caused by a rapidly changing magnetic field at $25 \mathrm{~Hz}$ [7]. The surface of the alumina ceramics is coated with titanium nitride (TiN) to suppress the secondary electron emission. Titanium is also used for flanges for the alumina ceramics beam pipes. In situ baking is not performed because the thermal expansion during baking may break the alumina ceramics due to an unacceptably large inner stress from the nearby metal parts. Turbomolecular pumps (TMPs) with the pumping speed of $1.3 \mathrm{~m}^{3} \mathrm{~s}^{-1}$ and ion pumps with the pumping speed of $0.8 \mathrm{~m}^{3} \mathrm{~s}^{-1}$ are installed for the main pumping system.

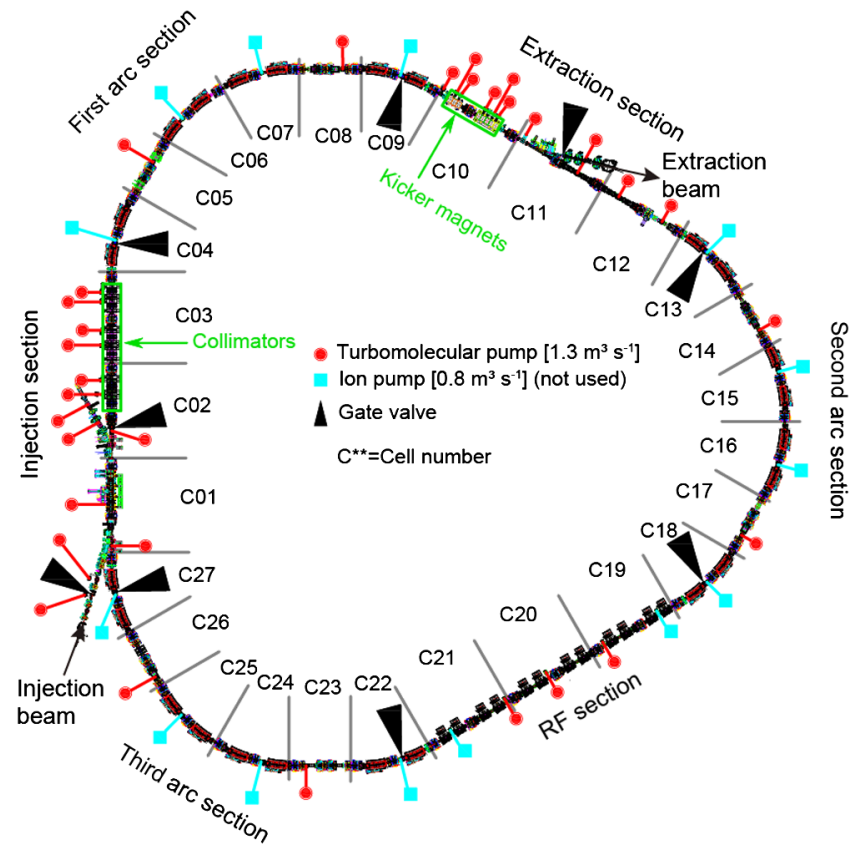

FIG. 1. Layout of the RCS vacuum system. The RCS consists of three straight sections and three arc sections, between which all-metal gate valves are installed. The beam line is divided into 27 cells for convenience. Vacuum gauges are not drawn in the figure. The ion pumps have not been used because of the intermittent outgassing problem (Fig. 4). The beam pipes and bellows are made of titanium. The beam pipes in the magnets are made of alumina ceramics, whose inner surface is coated by TiN.

The criterion of beam line pressure is considered as follows. The mean free path of the proton $\lambda$ is written as

$$
\lambda=\frac{1}{\sigma_{\mathrm{loss}} n_{\mathrm{gas}}},
$$

where $\sigma_{\text {loss }}$ is the cross section of beam loss by the Coulomb scattering and $n_{\text {gas }}$ is the density of the gas molecules in the beam line. The beam survival rate is estimated by the ratio of the mean free path and the total orbit length of the beam from the injection to the extraction. Even when the average pressure is $10^{-4} \mathrm{~Pa}$, the survival rate is estimated to be more than $99.99 \%$. However, we have set the pressure criterion to less than a low value in the $10^{-6} \mathrm{~Pa}$ order because a dynamic pressure by the beam is unpredictable. Figure 2 shows the typical pressure distribution of the beam line without beam [8]. The pressure measured by cold cathode gauges (CCGs) is plotted.

Radiation-resistant TMP is used as the main vacuum pumps to satisfy the former design concept. Such a TMP has been developed by changing certain parts that are not resistant to radiation (e.g., fluoroelastomer O-ring seals, fluorine resin sheath of wires, and epoxy resin insulators) with those that are radiation resistant (e.g., metal seals, polyether ether ketone (PEEK) sheath, and alumina 


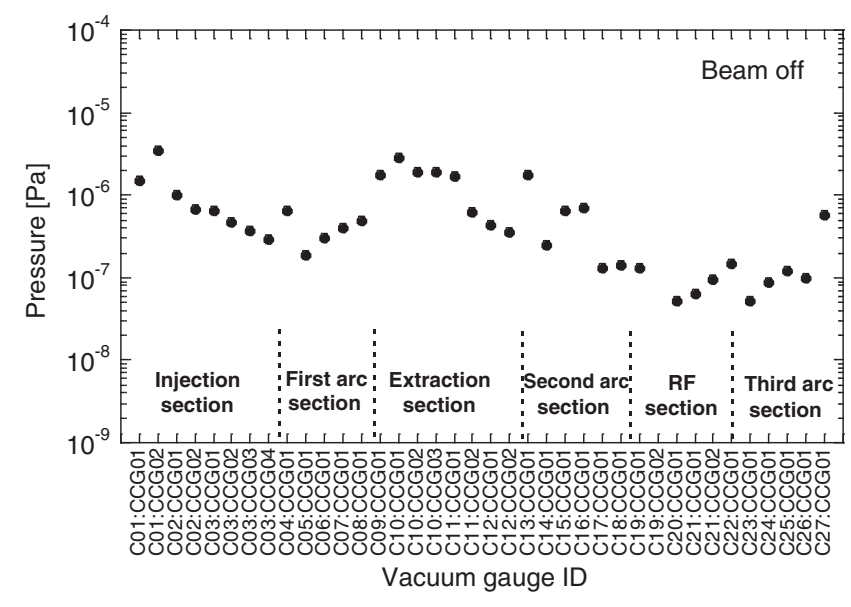

FIG. 2. Typical example of the beam line pressure distribution in the RCS. The pressure at about one month from the start of evacuation after the vacuum system shutdown.

ceramics insulators) $[9,10]$. The radiation-resistant TMP can operate with no trouble until the gamma-ray radiation dosage reaches over 70 MGy. PEEK is also used in the cables and connectors for control or power as insulator material [11]. Figure 3 shows the configuration diagram of the TMP system. The radiation-resistant TMP is installed in the accelerator tunnel. However, because the TMP controller uses semiconductor parts, it would easily be

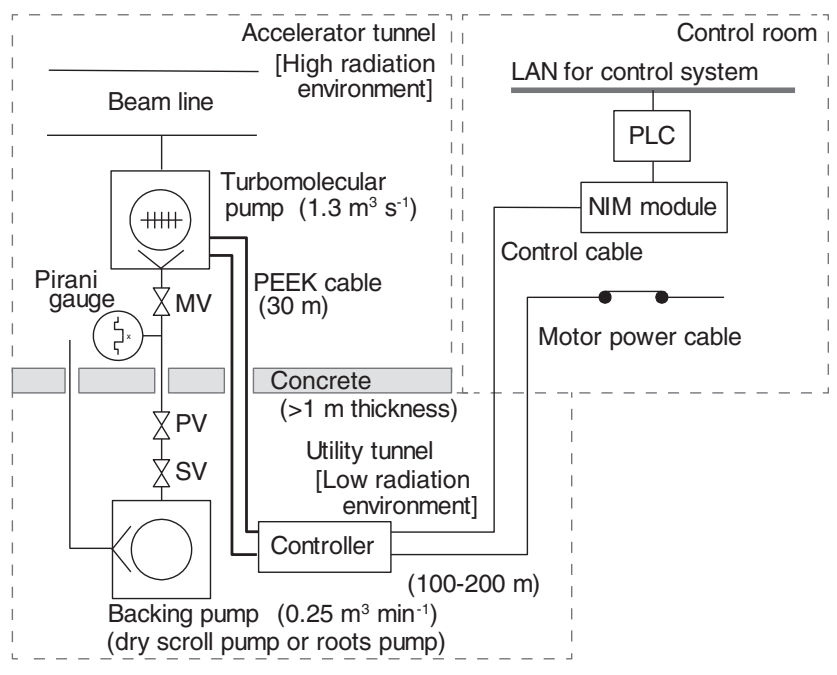

FIG. 3. Configuration of a TMP system. The radiation-resistant TMP is installed in the accelerator tunnel. The TMP controller and the backing pump, which are not radiation resistant, are installed in the utility tunnel. The accelerator tunnel and utility tunnel are separated by the concrete floor with a thickness of more than $1 \mathrm{~m}$. Thus, the radiation is much lower in the utility tunnel compared to the accelerator tunnel. There are three types of valves between the TMP and the backing pump, which are metal manual valve (MV), pneumatic valve (PV), and solenoid valve (SV). The SV closes when the electrical voltage is not applied to the backing pump. deteriorated or broken by radiation. Thus, the TMP controller is installed in the utility tunnel where the radiation is much lower compared to the accelerator tunnel because they are separated by ferroconcrete with a thickness of more than $1 \mathrm{~m}$. A dry scroll pump or roots pump is used for the backing pump of the TMP. The backing pump is also installed in the utility tunnel because a dry scroll pump has chip seals of a fluorine resin and a roots pump has an inverter circuit. In other words, the TMP system has been constructed such that the radiation-resistant and nonradiation-resistant equipment are arranged in appropriate places to prevent the system from deteriorating or failing due to radiation.

The pumping speed of the TMP is generally constant in a wide pressure range from around $10^{-1} \mathrm{~Pa}$ to $10^{-7} \mathrm{~Pa}$ [12]. Thus, the TMP can be effectively used for high-power beam accelerators like the RCS, where unexpected additional outgassing is caused by the beam. As shown in Fig. 1, a total of 32 TMP systems are currently installed in the RCS. The RCS vacuum system has been originally designed to simultaneously operate both the TMPs and the ion pumps. However, the intermittent pressure instability has occurred due to the ion pump operation. Figure 4 shows the pressure instability detected by the CCG in C19 as a representative. When the ion pumps are stopped, this phenomenon doesn't occur. Thus the gas is released from the ion pumps. The reason is considered that the ionized residual gas molecules are embedded in the cathode of the ion pump, then released after saturation. Therefore, ion pumps have not been used anymore to prevent the risk of interruption of the beam operation.

\section{B. Vacuum phenomena caused by the high-power beam}

So far, for more than 10 years, we have accumulated experiences related to the effects of a high-power beam

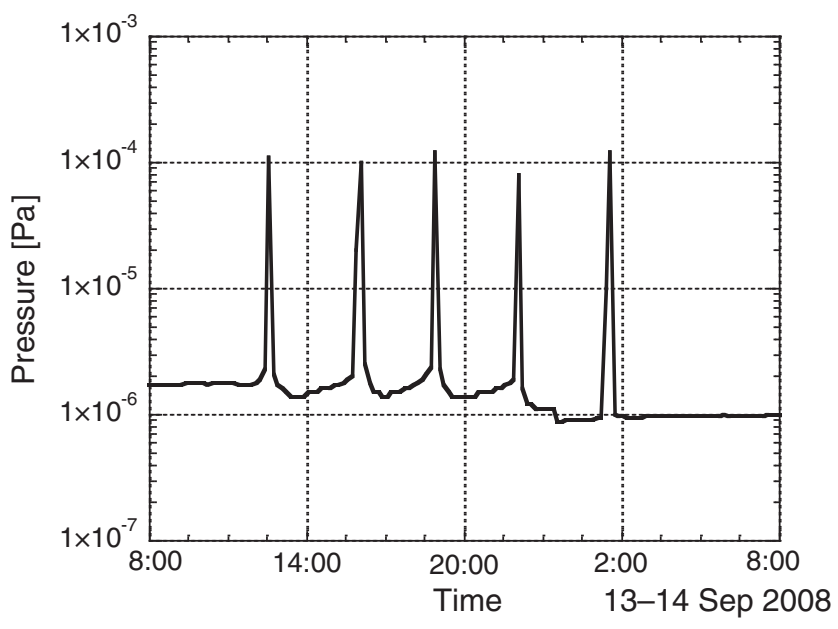

FIG. 4. Pressure instability caused by the ion pumps. The pressure measured by the CCG in C19 is plotted as a representative. 


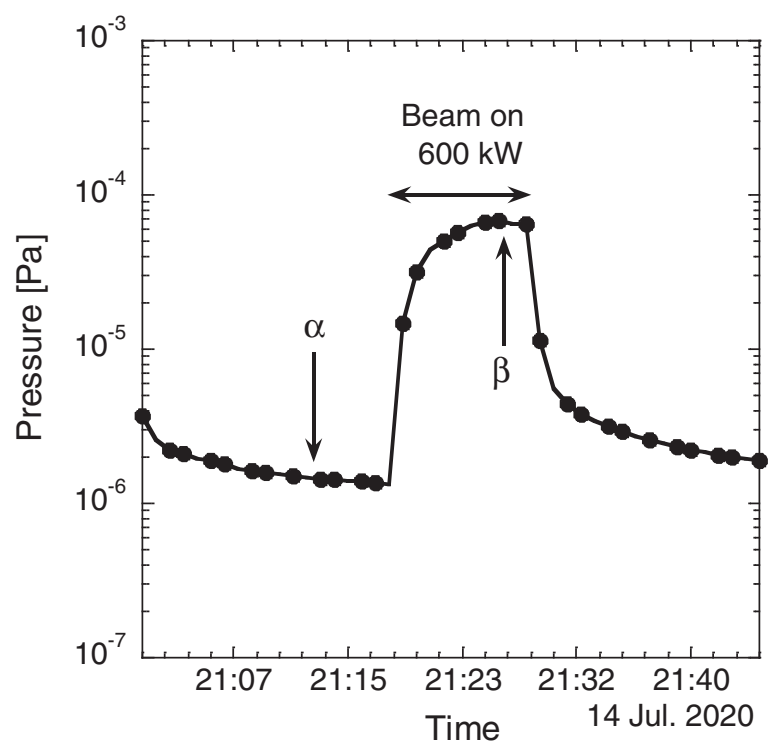

FIG. 5. Example of pressure rise during high-power beam operation. Pressure evolution during the beam commissioning with an output beam power of $600 \mathrm{~kW}$ is plotted. The pressure measured by a CCG in $\mathrm{C} 15$ of the second arc section is shown in this figure as a typical case.

operation. Consequently, it is clarified that the high-power beam operation has more impacts on the vacuum system than expected at the time of the design. The phenomena caused by the high-power beam are divided into the following two categories: (1) malfunction of the vacuum equipment because of high-intensity beam loss and (2) a large increase in the beam line pressure during high-power beam operation.

A specific example of the former category is the TMP system failure caused by the full-beam loss in the acceleration test of the high-intensity beam. During the beam commissioning, a single-shot beam is used to search for parameters with beam loss as low as possible (e.g., excitation pattern of the magnets, RF acceleration pattern, feedback pattern, etc.) [3]. As mentioned above, the beam repetition rate is $25 \mathrm{~Hz}$ under the normal operation. A single-shot beam means that the single-pulse beam is injected, accelerated, and extracted without repetition. The corresponding beam power when the same number of particles in a single-shot pulse operates at the rated repetition rate of $25 \mathrm{~Hz}$ is called the equivalent beam power. In recent years, the RCS has tested the single shots with an equivalent beam power of more than $1 \mathrm{MW}$. The current acceleration cavity has been designed to accelerate to a maximum beam power of $1 \mathrm{MW}$. Thus, in such a highintensity shot with an equivalent beam power of more than $1 \mathrm{MW}$, the beam has been fully lost in the midway of the acceleration [13]. During such beam commissioning in the year 2018, the TMP system has failed at the same timing as a single-shot beam of the 1.2 MW equivalent power. This event requires a review of the above-described precondition
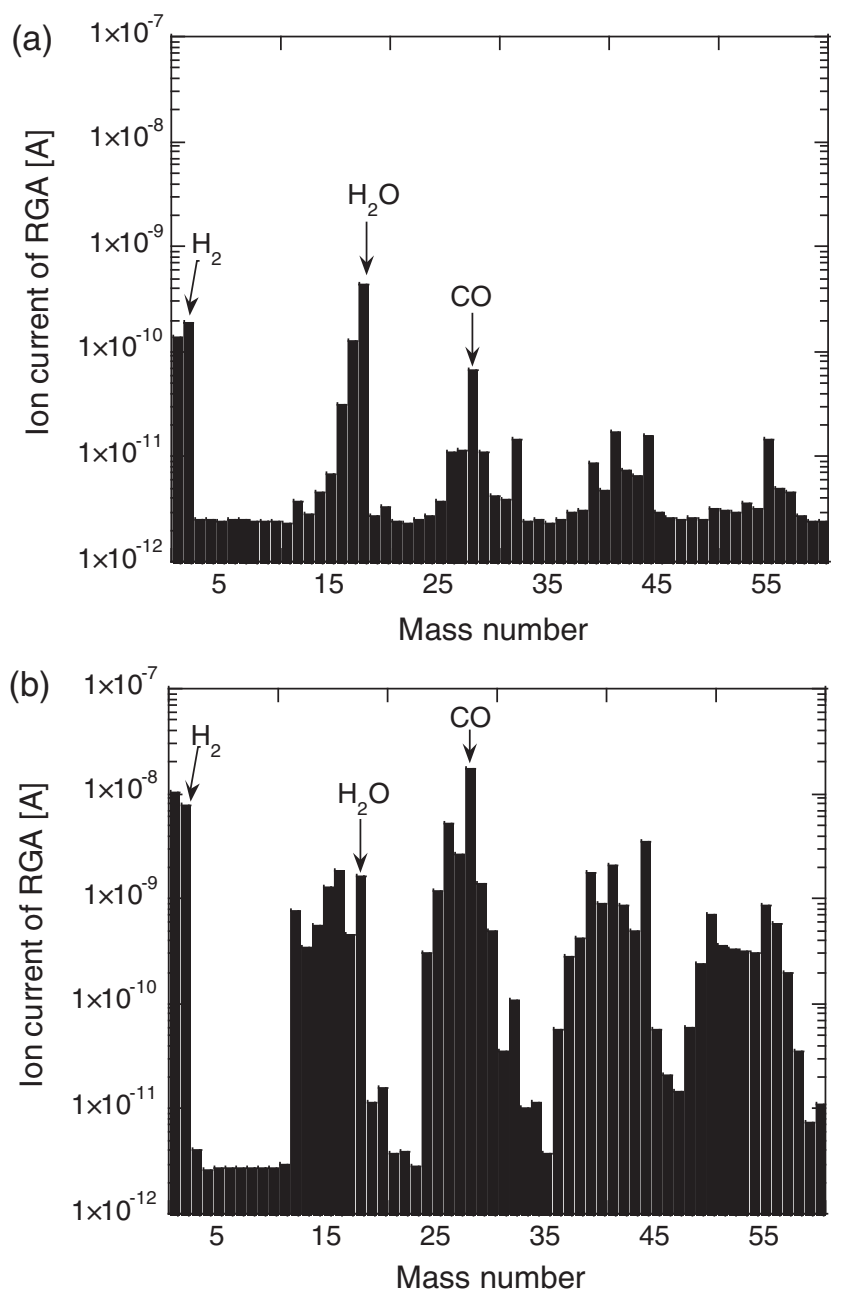

FIG. 6. Example of residual gas spectra in the beam line (a) without and (b) with beam measured by the RGA in the second arc section. Each spectrum corresponds to points $\alpha$ and $\beta$ in Fig. 5, respectively.

that the TMP system is constructed to prevent the system from failing due to radiation.

As the latter category of the phenomena caused by the high-power beam, an extreme beam line pressure rise has been often observed so far during the high-power beam operations. Figure 5 shows one of the examples. In this case, a beam line pressure rises by nearly two orders of magnitude in the beam operation with an output beam power of $600 \mathrm{~kW}$. Figures 6(a) and 6(b) show the spectra of the residual gas analyzer (RGA) with and without $600 \mathrm{~kW}$ beam, respectively. It is noticed that carbon monoxide is the main outgassing component during the beam operation, while water is the main residual gas without the beam.

An extreme beam line pressure rise with an increasing beam current (or beam power) is called the "pressure runaway" [14]. The pressure runaway has been first historically observed in the intersection storage ring (ISR) in CERN $[15,16]$. In the ISR, $28 \mathrm{GeV}$ protons are accumulated in two counter circulating orbits. The average 


\section{(a)}

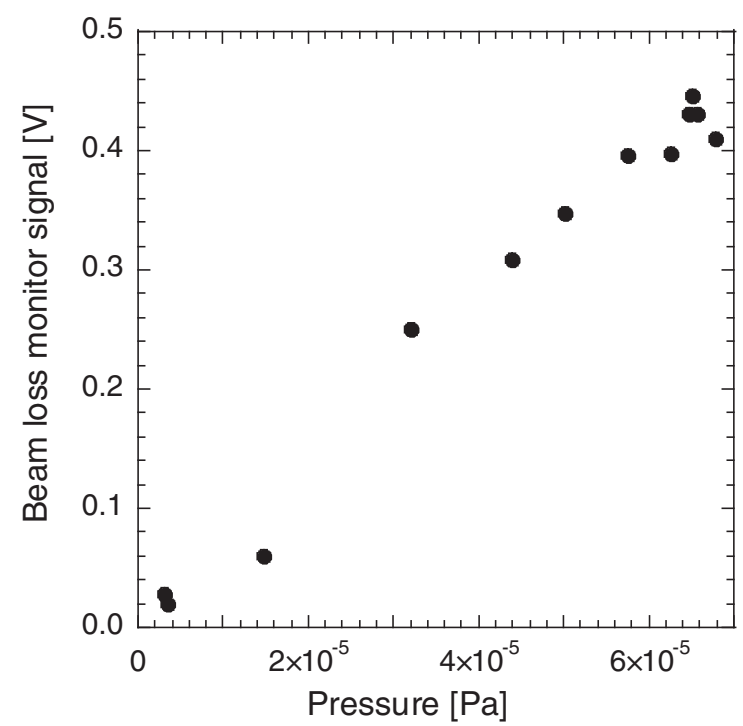

(b)

(b)
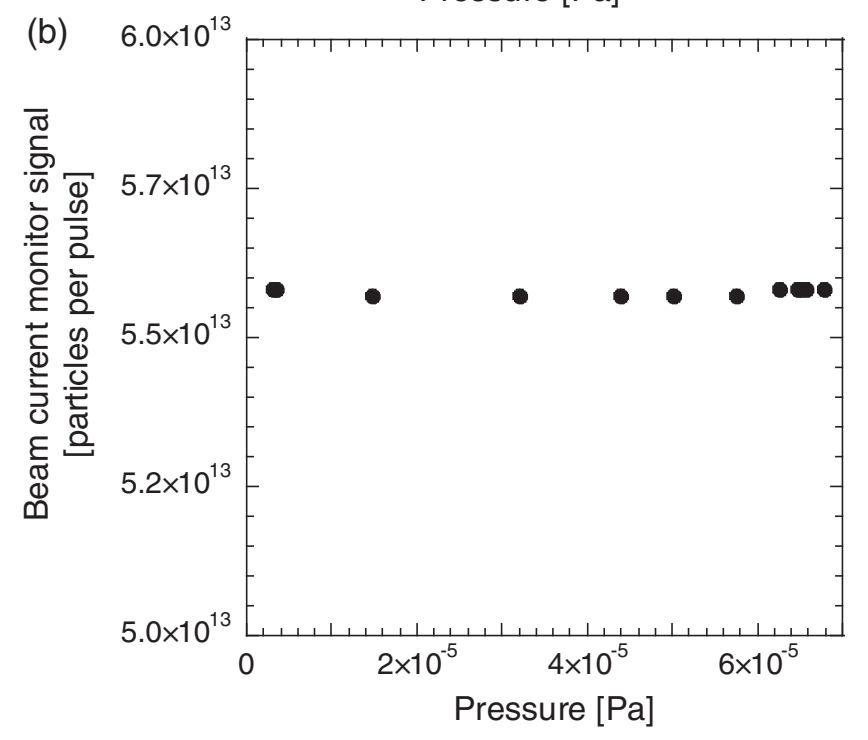

FIG. 7. (a) Example of the correlation between the beam line pressure and the beam loss monitor signal in the $600 \mathrm{~kW}$ beam operation of Fig. 5. (b) Example of the correlation between the pressure and the number of protons measured by the beam current monitor in the same period as panel (a).

beam line pressure is $10^{-8} \mathrm{~Pa}$ without beam. The problem has occurred when they started a high-intensity beam accumulation. With increasing the beam current by accumulating the beam pulses, the catastrophic pressure increase by a few orders of magnitude has occurred. Consequently, the beam current has decayed rapidly. The cause is thought to be the scattering by the gas nuclei and beam neutralization by the electrons created in the ionizing events [17]. In the RCS case, the relation between the beam current and the pressure rise is different from the ISR case. Figure 7(a) shows the relation between the pressure and the beam loss monitor signal. Figure 7(b) shows the relation between the pressure and the beam current monitor signal. The beam loss monitor signal proportionally increases with the pressure. On the other hand, the beam current monitor signal doesn't decrease even when the pressure increased. One reason is that the beam loss is too small to be detected as the signal reduction of the beam current monitor. Another reason is that the increase of the beam loss monitor signal doesn't originate from the real beam loss. The possible candidate of such false beam loss signal increase is the $\mathrm{x}$ ray released when the molecules are ionized by the beam, the bremsstrahlung from the ions and electrons when they are accelerated by the beam potential, etc. Anyway, the pressure rise interrupts the beam operation because the increase of the beam loss monitor signal stops the beam by the machine protection system when it exceeds the threshold value. Therefore, for future stable user operations with the high-power beam, understanding the dynamic pressure behaviors, clarifying the mechanism of the dynamic pressure, and undertaking adequate vacuum system improvements are indispensable.

This paper reports the improved vacuum system for the high-power beam operation in the RCS from two perspectives: (1) Improved TMP system to prevent the failure caused by the high-intensity beam loss and (2) establishment of the pressure runaway suppression method through an understanding of the dynamic pressure mechanism. In Sec. II, we describe the failure event of the TMP caused by the high-intensity beam loss. The countermeasure against such TMP failure is explained in Sec. III. The subsequent sections describe the pressure runaway during the highpower beam operation. In Sec. IV, the typical dynamic pressure behaviors are systematically investigated. Besides, the dynamic pressure mechanism is verified by comparing the measurement with the analytic calculation. The critical parameters for the pressure runaway are also elucidated by the calculation. Finally, in Sec. V, we describe the improved vacuum system to suppress the pressure runaway.

\section{TMP FAILURE BY HIGH-INTENSITY BEAM LOSS}

Figure 8 shows the beam loss monitor signal distribution when a single-shot beam with the beam power equivalent to 1.2 MW is fully lost. The TMP system in C14 of the second arc section failed at the same timing as the single shot. The vertical axis denotes the signal of the beam loss monitors. The signal is integrated for $20 \mathrm{~ms}$, which is one cycle from the beam injection to the extraction. Figure 8 also includes the beam loss signal distribution in the normal $1 \mathrm{MW}$ beam acceleration for reference. A comparison of these distributions shows that the amount of the beam loss when the TMP trouble occurred is more than 20 times larger than the normal case on average. The beam loss around C14 is the maximum. The TMP controller of C14 in the utility tunnel has been broken without error output. An investigation of the failed controller has revealed that the digital signal processor is defective. The TMP has made a rubbing noise when it restarts with a replaced controller. Then, the TMP 


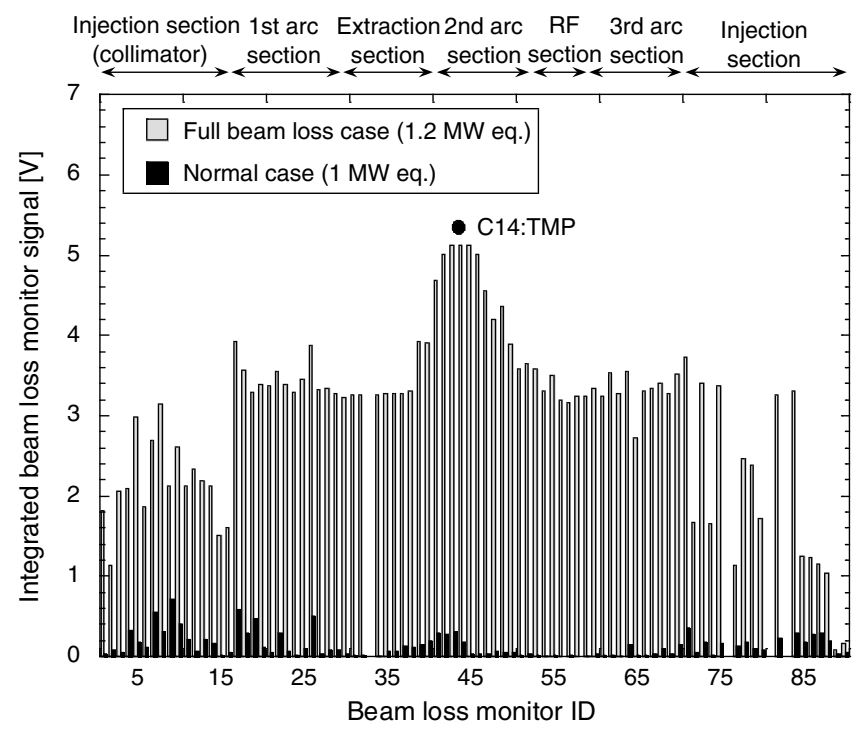

FIG. 8. Beam loss monitor signal distribution when a singleshot beam with the power equivalent to $1.2 \mathrm{MW}$ is fully lost. The signal is integrated for one cycle from the beam injection to the extraction, $20 \mathrm{~ms}$. The position of the failed TMP system is also shown. The beam loss signal distribution in the normal $1 \mathrm{MW}$ equivalent beam acceleration case is also shown for reference.

has stopped by the rotor displacement error before it reaches the rated rotation speed of $560 \mathrm{~Hz}$. Figure 9 shows the deformed rotor and stator blade of the TMP. Figure 10 depicts the damaged inner rail and ball of the touch-down
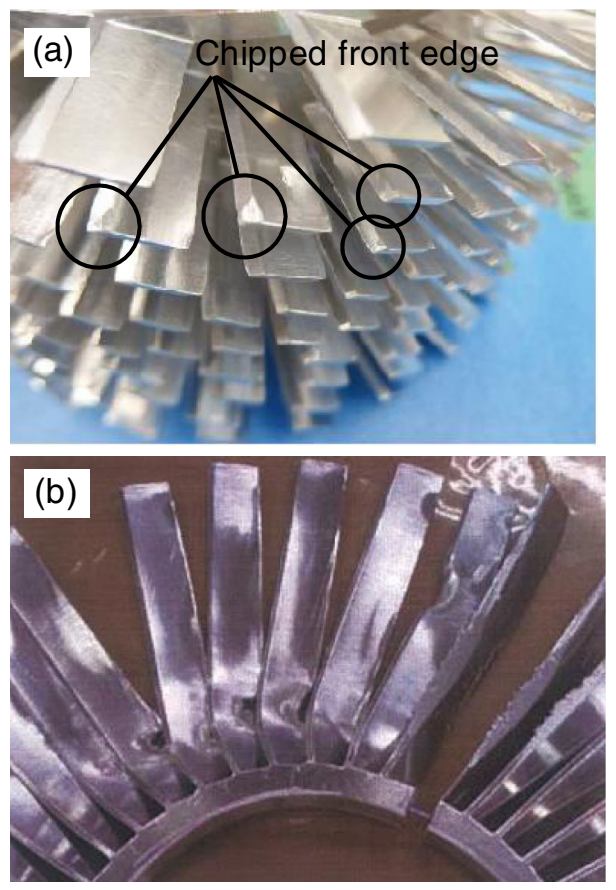

FIG. 9. Damaged rotor and stator blade of the TMP. The diameter of the rotor and stator is about $220 \mathrm{~mm}$. (a) Chipped edges of the rotor. (b) Deformed stator blade.

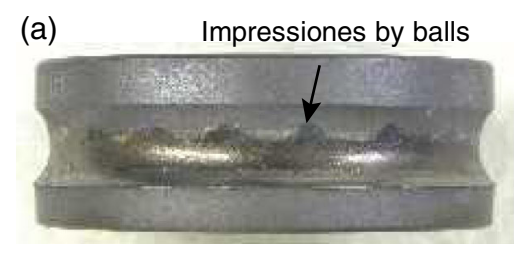

(b)

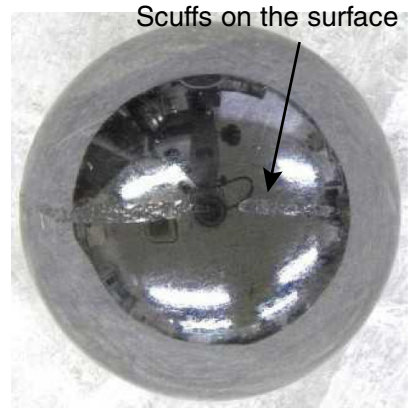

FIG. 10. Damaged bearing parts of the TMP. (a) Inner bearing rail with impressions by the balls. (b) Bearing ball with scuffs.

bearing. Impressions caused by the balls are found on the rail and the ball surface has large scratches. From these facts, the failure is considered to have occurred as follows: (1) a high-intensity single-shot beam is fully lost with a peak in the vicinity of $\mathrm{C} 14,(2)$ the high-energy secondary particles pass through more than $1 \mathrm{~m}$ thick concrete floor of the accelerator tunnel and destroy the digital signal processor in the controller, which is located in the utility tunnel (Fig. 3), (3) the control signal and the motor power from the controller are lost. The rotor is touched down from the magnetic levitation with a rated rotational speed. The brake is not applied after touch down because the controller has been broken. We define this event as a free-run touch down (FRTD) as distinguished from normal touch down with brake, and (4) a large load by the bearing ball compresses the bearing rail by more than $1 \mathrm{~mm}$. The normal gap spacing between the rotor and stator blade is $1 \mathrm{~mm}$. Consequently, the rotor touches the stator blade, and they are deformed.

In a normal touch-down case, the rotor is immediately decelerated by an electromagnetic brake after the touch down. In the case of an electrical power loss, the power is generated to the controller by the rotation of the rotor, and then the rotor is decelerated to $30 \%$ of the rated rotational speed and the magnetic levitation is stopped. In those cases, the load to the bearing is much smaller compared to the FRTD case. The manufacturer makes sure that there are no problems after more than 10 times normal touch downs or 100 times power loss touch downs. We've had similar experiences in the past where the TMP is broken by the FRTD due to controller failure. In past cases, the controller failure has been caused by electrical noise from the power line of the pulse magnets. The past events and a countermeasure are briefly reported in another article [8]. The same countermeasure is applied to this case. Therefore, the 


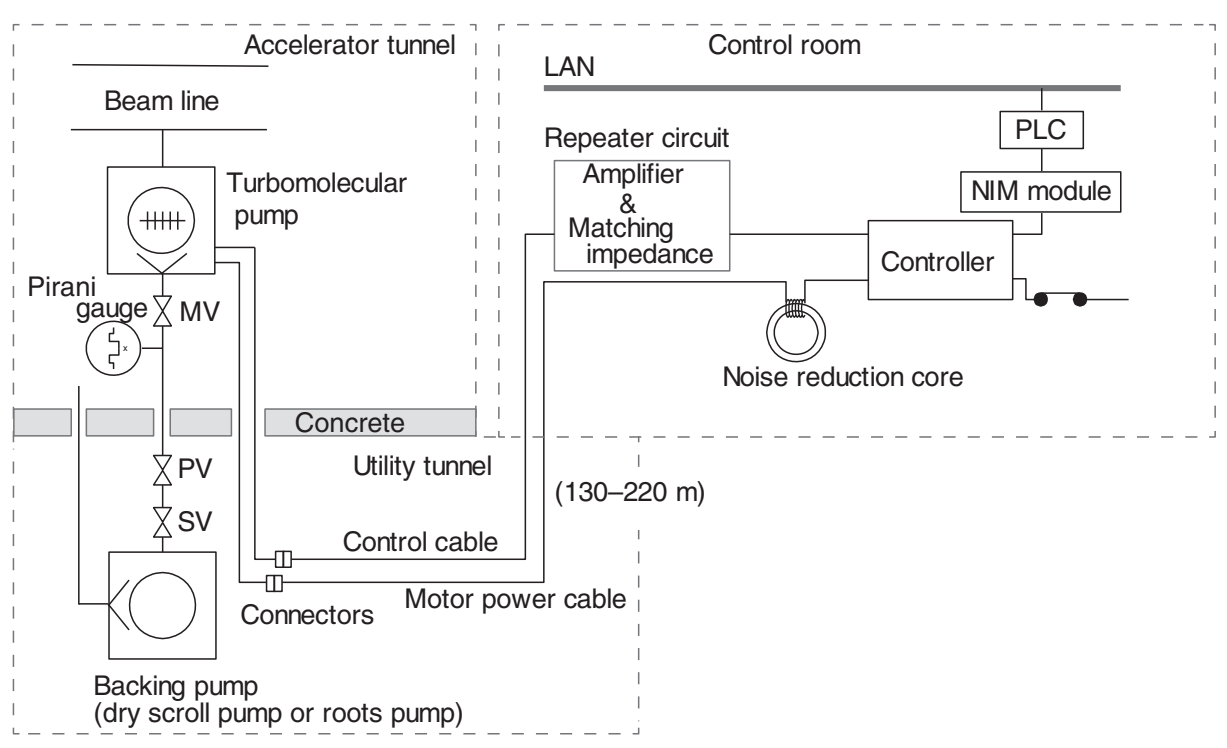

FIG. 11. Configuration diagram of the improved TMP system using the controller with the long cable specification. The controller is $220 \mathrm{~m}$ away from the TMP in maximum. There is no radiation influence in the control room.

following content is partially overlapped with the past article. However, more details are described in the next section.

\section{IMPROVED TMP SYSTEM}

The following two countermeasures are taken against TMP system failure: (1) development of a TMP controller that can use long motor and control cables to install the controller in a distant area where there is no radiation influence and (2) development of a TMP with an improved bearing more tolerant for FRTD.

The former is a fundamental measure for preventing the controller from failing by radiation, while the latter aims to enable the TMP to restart even when the FRTD occurs.

\section{A. TMP controller with long cable specification}

The standard cable length between TMP and controller is $30 \mathrm{~m}$ in maximum. The revised controller has been developed to operate with a longer distance between the TMP and the controller. The cable is lengthened to reduce the radiation damage to the controller by installing it in the control room. Figure 11 shows the improved configuration diagram of the TMP system using the controller with the long cable specification. The maximum distance between the TMP in the accelerator tunnel and the controller in the control room is $220 \mathrm{~m}$. It is possible to enter the control room even during beam operation. Thus, another advantage of the long cable specification is that the TMP status can always be checked by the controller's panel even during the beam operation.

There are two types of cables between the TMP and the controller, which are used for the rotor position control and motor power, respectively. The control cable includes the lines for the control signals from the coil sensors for detecting the rotor position and rotation speed. The maximum length of the cables is limited by the attenuation of the signal amplitude from the coil sensor. The repeater circuit which included the amplifier is installed near the controller to increase the cable length. The repeater circuit needs to include the matching impedance parts. The total impedance of the TMP and the cable depends not only on the cable length but also on the floating capacitance and the inductance varied by the cable laying environment. Therefore, the resistances and the capacitors have to be individually changed to match the impedance after laying the cables such that a rotor can accelerate up to the rated rotation speed without displacement error. The maximum cable length has been examined with Osaka Vacuum, Ltd., Osaka, Japan [18], on the cases of vertical and horizontal TMP mounting. Consequently, the maximum cable length is 230 and $180 \mathrm{~m}$ for the vertical and horizontal mounting, respectively. The control cable also includes the electromagnet power lines for the rotor position control. The cable length of the vertical mounting is longer than that for the horizontal mounting because it uses less power for the rotor positioning control due to the gyro effect that stabilized the rotational body.

The electrical noise released by the inverter circuit inside makes the problem when the controller is operated in the control room. The electrical noise causes the Nuclear Instrumentation Module (NIM) modules of the other vacuum devices in the same control rack to malfunction. A noise reduction core is inserted into the motor power line (Fig. 11). The core material and the winding number have been optimized. Consequently, the NIM module malfunction is prevented by winding the motor cable 50 turns on a FINEMET $^{\circledR}$ common mode choke core with $79 \mathrm{~mm}$ outer diameter, $51 \mathrm{~mm}$ inner diameter, and $25 \mathrm{~mm}$ thickness [19]. 
TABLE II. Measured surface roughness and hardness of the bearing parts. The measured values of the bearing parts unused and after the FRTD are presented for the normal and improved ones, respectively.

\begin{tabular}{lccccc}
\hline \hline & $\begin{array}{c}\text { Unused normal } \\
\text { parts }\end{array}$ & $\begin{array}{c}\text { Normal parts } \\
\text { after FRTD }\end{array}$ & $\begin{array}{c}\text { Unused } \\
\text { improved parts }\end{array}$ & $\begin{array}{c}\text { Improved parts } \\
\text { after FRTD }\end{array}$ \\
\hline Inner bearing rail & Surface roughness: $\mathrm{Ra}[\mu \mathrm{m}]$ & 0.02 & 0.6 & 0.02 & 0.1 \\
& Vickers hardness: $[\mathrm{HV}]$ & 720 & 580 & 850 & 830 \\
Bearing ball & Surface roughness: $\mathrm{Ra}[\mu \mathrm{m}]$ & 0.01 & 0.5 & 0.01 & 0.1 \\
\hline \hline
\end{tabular}

\section{B. TMP tolerant to FRTD}

As shown in Fig. 10, the rails and the balls of the TMP bearing have serious damages by the FRTD. Table II summarizes the measured surface roughness and hardness of the normal bearing parts unused and after the FRTD. The roundness distribution of the normal inner bearing rail after the FRTD is shown in panel (a) of Fig. 12. The results imply that the FRTD causes the large deformation and hardness reduction of the normal bearing parts.

The bearing has been improved step by step in response to the results of the repeatedly performed FRTD tests. The final improved items are listed: (1) the number of bearing balls is decreased to prevent contact between the balls, (2) the ball with a smaller diameter is used to reduce the centrifugal force for decreasing the stress on the outer rail. Besides, the rail shape is changed to increase the contact area for the balls to decrease the stress on the rail, and (3) the rail material is changed from stainless steel to tool steel with a high degree of hardness.

The roughness and hardness of the improved bearing parts unused and after the FRTD test are presented in Table II. The roundness of the improved bearing parts after the FRTD test is shown in panel (b) of Fig. 12. It is found that there are little deformation and hardness reduction with the improved bearing parts after the FRTD test. Figure 13 shows the typical result of the FRTD test of the TMP with the normal and improved bearing. It represents the temporal variation of the rotation speed of the rotor from the FRTD. The rotation speed of the rotor rapidly decreases for the normal-type bearing because of the increased friction. In contrast, the rotation speed of the rotor with the improved bearing smoothly decreases. The TMP with the improved bearing can restart after more than four times of FRTD. The usual touch-down test, in which the brake is applied to the rotor, has been also performed. The result shows that the TMP with the improved bearing can be operated even after more than 20 times the usual touch down.

The TMP system becomes more tolerant of the large loss of the high-intensity beam using the controller with the long cable specification and the TMP with the improved bearing. However, the roots pump used as the backing pumps still has the potential risk for failures by the large beam loss because it has an inverter circuit inside. We plan to separate the power supply from the roots pump body.
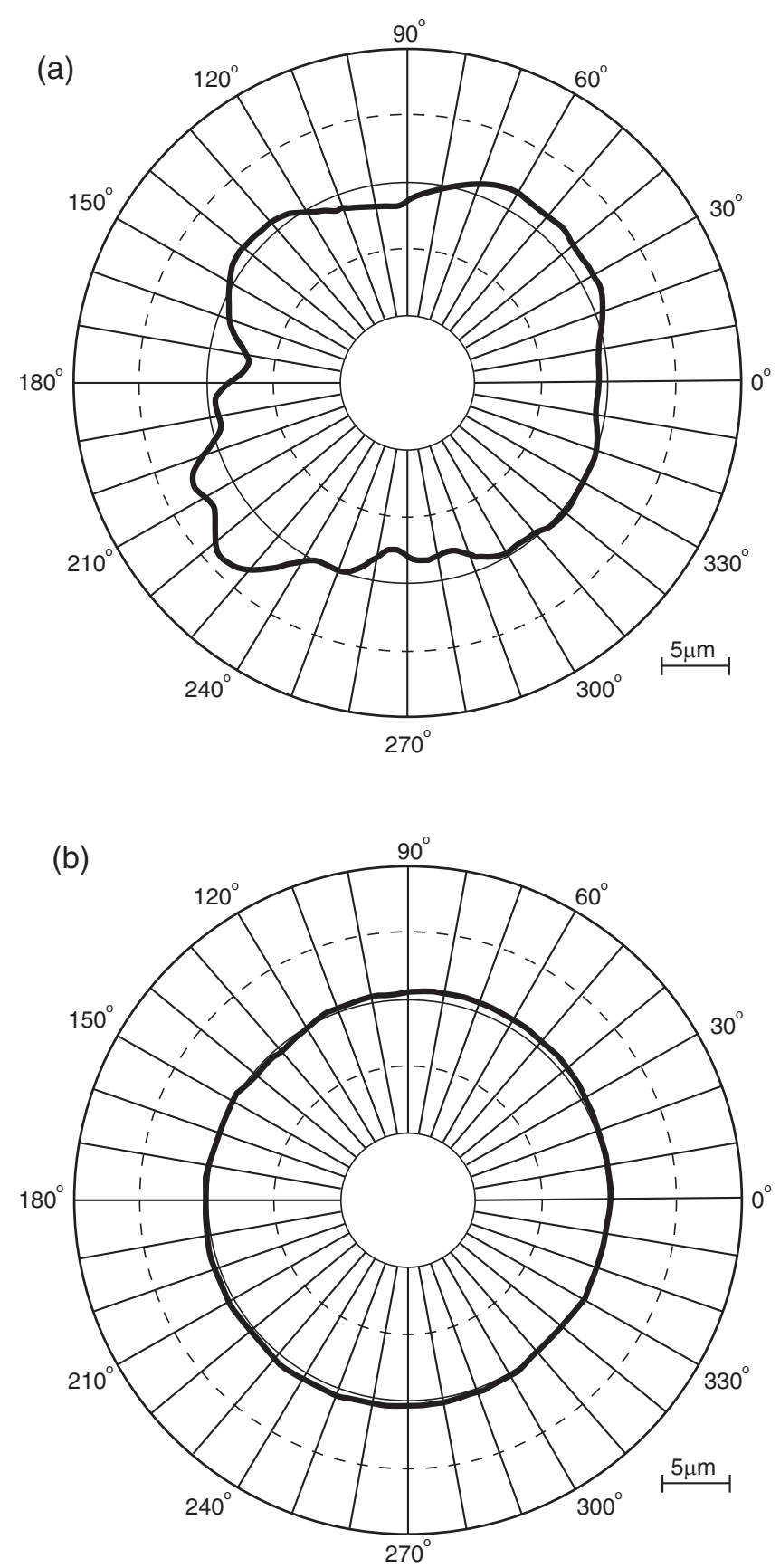

FIG. 12. Measured roundness distribution of the inner bearing rail after the FRTD test for the (a) normal and (b) improved bearings. 


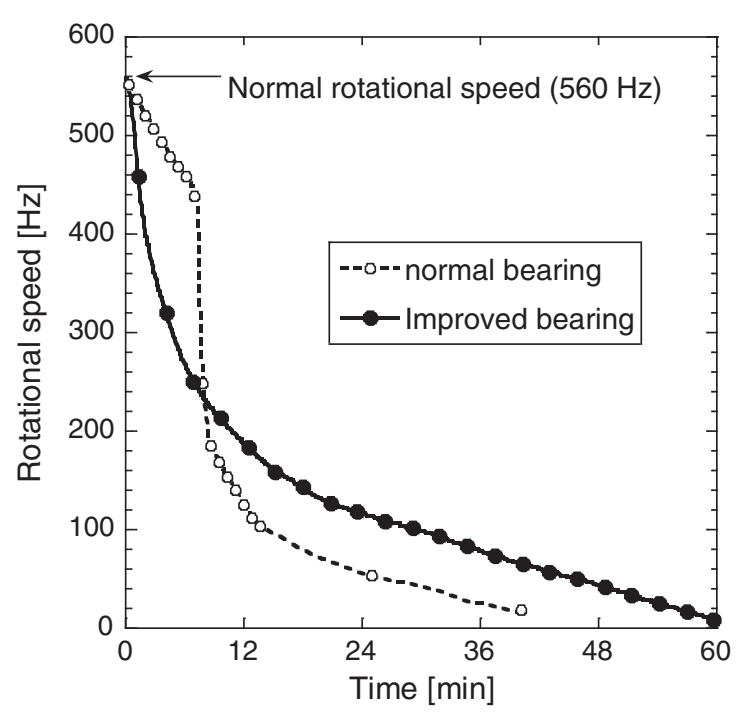

FIG. 13. Change of the rotor rotation speed against the time in the FRTD test for the TMP with the normal bearing and improved one. The origin of the horizontal axis is the time of the rotor touch down.

And the power supply with the long cable specification will be installed in the control room based on the same idea as the TMP controller.

\section{DYNAMIC PRESSURE IN BEAM OPERATION}

\section{A. Measured dynamic pressure}

Figure 14 shows the history of the output beam power from the RCS to the MLF. The typical behaviors of the beam line pressure at some points from the year 2014 to 2015 are summarized in Fig. 15. Each panel (a) to (e) in Fig. 15 corresponds to points A to E in Fig. 14, respectively.

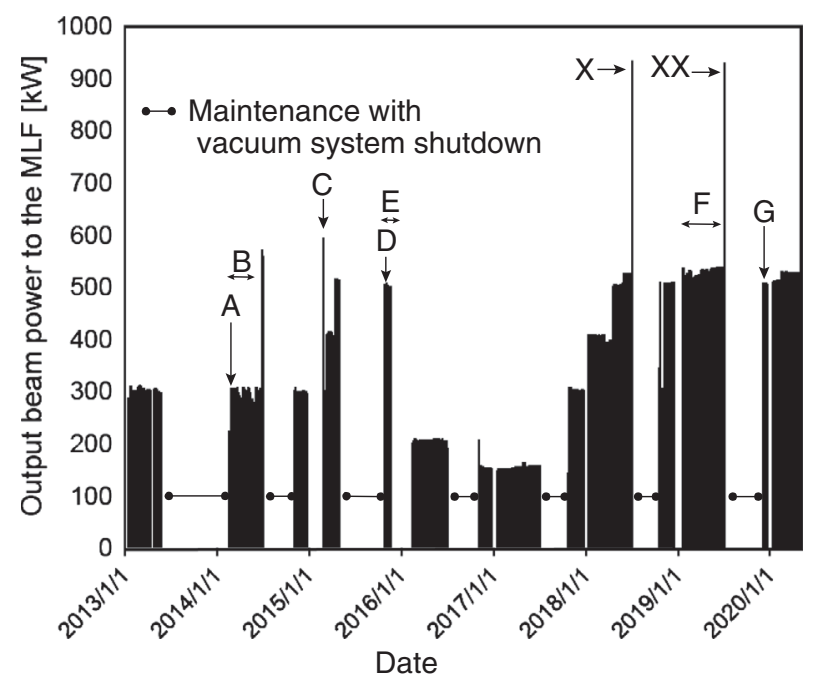

FIG. 14. History of the output beam power to the MLF from the RCS. Denotations A-G, X, and XX represent points, for which the dynamic pressures are analyzed in the present work.
The pressure measured by the representative CCG in each section is plotted. The situation of points $A$ to $E$ is described:

(Point A) The time of the beam commissioning after position alignment work of the beam line equipment, such as the magnets and beam pipes. The equipment position has displaced because of the Great East Japan Earthquake in 2011. All the beam lines have been purged by argon gas to atmospheric pressure in the alignment work. Almost all the beam line flanges have been opened to air after removing the clamps. The flanges have been covered by plastic sheets and rubber caps to prevent the dust from entering inside the beam pipes. The alignment work has taken six months. In the beam commissioning after the alignment work, a large pressure rise by a few orders of magnitude occurs with the $300 \mathrm{~kW}$ beam power [Fig. 15(a)].

(Point B) The period of the user operation with the beam power of $200-300 \mathrm{~kW}$ after point A. The beam line pressure rise gradually decreases during the continuous beam operation [Fig. 15(b)].

(Point C) The time of the beam commissioning after the beam operation suspension for two months. The beam line has kept being evacuated by the TMPs during the suspension. The pressure rise by more than an order of magnitude doesn't occur, even though the beam power is larger than that in point A [Fig. 15(c)].

(Point D) The time of the beam commissioning after the vacuum system shutdown for maintenance. The vacuum system has been stopped during the maintenance period. In the injection, first arc, extraction, and RF sections, the beam line has been purged to atmospheric pressure by argon gas, and only a few flanges in each section have been opened to air for the vacuum gauge exchange or TMP maintenances. The flanges have been opened for a few hours to 1 day. Other sections have been kept in a vacuum with the TMPs stopped and with the pneumatic valve (PV) in Figs. 3 and 11, closed. The amount of pressure rise is larger than that in point $\mathrm{C}$ despite the similar beam power, while it is much smaller than that in point A [Fig. 15(d)].

(Point E) The period of user operation with $500 \mathrm{~kW}$ beam power after point $\mathrm{D}$. The gradual decreasing trend of the pressure rise during the beam operation is similar to that in point B [Fig. 15(e)].

Figure 16 shows the dependence of the pressure on the beam power at points A, C, and D. The CCG values in C06 and $\mathrm{C} 24$ are plotted as the representatives. The maximum pressure value at each beam power is normalized by the pressure without beam. The pressure is found to increase nonlinearly with the beam power in any case. The relation between the maximum pressure and the beam power is very different from each point. Especially in point A, which is after all the flanges have been opened for six months, the pressure runaway occurs in a relatively low-beam power of 

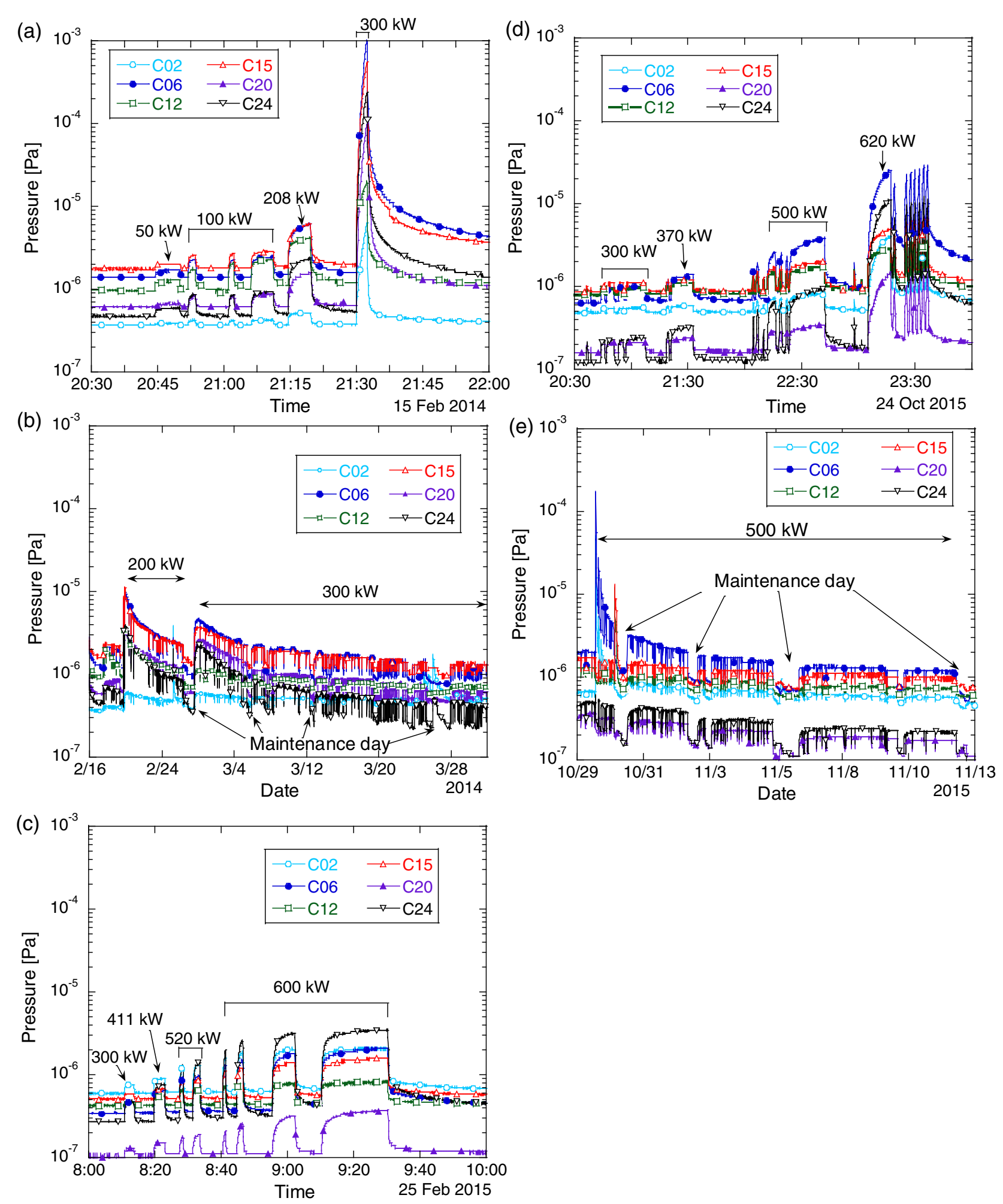

FIG. 15. Beam line pressure during the high-power beam operation. Panels (a) to (e) correspond to the beam line pressure in points A to E in Fig. 14, respectively. The pressure measured by the representative CCGs in each section is plotted. C02: Injection section, C06: first arc section, C12: Extraction section, C15: second arc section, C20: RF section, and C24: third arc section. (a) (Point A) Pressure in the beam commissioning after position alignment work of the beam line equipment. The beam lines in all sections have been purged by argon gas and opened to air during the work. (b) (Point B) pressure during the user operation at the 200-300 kW beam power after point A. (c) (Point C) pressure in the beam commissioning after suspension of the beam operation. The beam line has been kept to be evacuated by the TMPs during the suspension. (d) (Point D) pressure in the beam commissioning after vacuum system shutdown for maintenance. A few flanges in some sections have been opened to air for a short time. (e) (Point E) pressure during the user operation at the $500 \mathrm{~kW}$ beam power after point $\mathrm{D}$. 


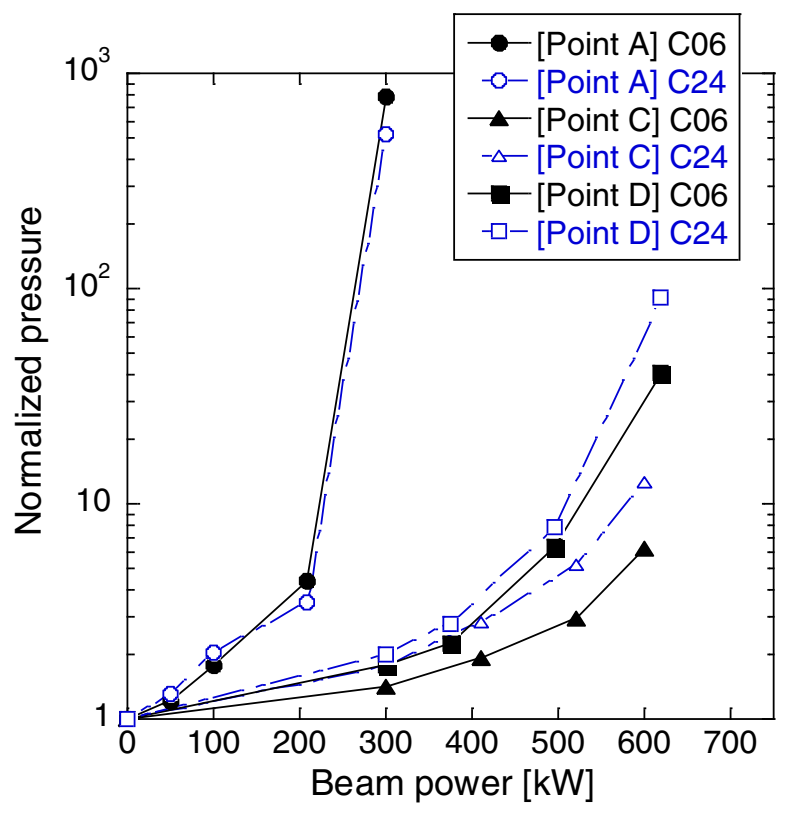

FIG. 16. Relation of the maximum pressure to each beam power in points A, C, and D of Fig. 14 [Figs. 15(a), 15(c), and 15(d)]. The CCG values in C06 and C24 are plotted as representatives. Each pressure value is normalized by the pressure without beam.

$300 \mathrm{~kW}$. The dynamic pressure becomes three orders of magnitude higher than the pressure without a beam. In point $\mathrm{C}$, which is after the beam suspension with the beam lines evacuated by the TMPs, the dynamic pressure is just about 10 times larger than the static pressure even in the higher beam power operation of $600 \mathrm{~kW}$. In point $\mathrm{D}$, which is after the maintenance with a few flanges opened in some sections for a short time, the amount of the pressure rise is between those of points A and C. As shown in Fig. 15(d) for point $\mathrm{D}$, there is no apparent larger pressure rise in the sections where the flanges have been opened to air than that in sections kept in a vacuum. The reason is considered that the number of open flanges is small and the time of the open to the air is short.

The relation between the beam operation and the beam line pressure can be summarized from these data as follows: (1) The pressure nonlinearly increases with increasing the beam power. Especially, an extreme pressure rise tends to occur after the beam line opening to air in the maintenance for a long period and (2) The conditioning effect, which represents the gradual decrease in the amount of pressure rise during the continuous beam operation, is observed.

Here, we consider the mechanism of the dynamic pressure in the RCS from the above characteristics and comparison with the other machines. The nonlinear pressure rise in the RCS with increasing beam power is similar to the ISR case [15-17]. The pressure runaway in the ISR is explained by the avalanche effect of the ion- stimulated gas desorption (ISD). That is, the residual gas molecules ionized by the beam are accelerated by the electrical potential between the beam bunch and the beampipe and then knock out the adsorbed molecules on the wall surface. The desorbed molecules are then ionized by the beam. The repetition of such a process generates an avalanche of molecules in the gas phase. As another case, the pressure in the electron and positron damping rings for the ILC is estimated as a function of the beam current in Ref. [14]. The pressure in the electron dumping ring almost proportionally increases with the beam current due to the increasing intensity of synchrotron radiation and the resulting photon-stimulated desorption. The situation is different in the positron dumping ring at high-beam current. As the beam current increases, the pressure in the positron ring steeply increases, and eventually, it runs away. The mechanism of this phenomenon in the positron ring is the ISD. The ions are accelerated by the positively charged beam to the wall and desorb the adsorbed molecules. Then, the desorbed molecules are ionized by the beam, the repetition of such a process generates the avalanche of the gas molecules, which is the same as the ISR case. On the other hand, the electron cloud could be the source of the dynamic pressure. Estimation of the amplification factor of the secondary emission electron cloud has been performed for some proton rings including the RCS [20,21]. The maximum secondary electron emission yield $\delta_{2, \max }$ of 2.1 is assumed in the calculation. They conclude that the bunched beams at the extraction of the RCS are near the instability threshold. They also describe that the surface treatment of the beam pipe to reduce $\delta_{2, \max }$ like TiN coating, can drastically suppress the electron cloud buildup. The usual $\delta_{2, \max }$ of the TiN coated surface is about 1.5 [22]. In the RCS, the inner surface of the alumina ceramics is coated by the TiN film. The other part is mainly titanium whose $\delta_{2, \max }$ is about 2 . The rate of the TiN coated surface area to the whole surface area in each section is about $40 \%, 30 \%$, and $40 \%$ for the injection, extraction, and RF section, respectively. The rate of the TiN coated surface is about $60 \%$ in the arc sections. Thus, the current RCS is under the threshold of electron cloud instability. Besides, there is no obvious correlation between the TiN coated surface rate and the measured pressure rise in each section as shown in Fig. 15. It is considered that the electron cloud is not the main source of the pressure rise in the RCS.

The relativistic heavy ion collider is one of the accelerators where the beam line pressure has risen due to the electron cloud-sourced gas desorption [23,24]. In the relativistic heavy ion collider, the tune shift, which is a linear function of the spatial density of the electron cloud, has been observed in the train of beam bunches [23]. They have also directory measured the electron in the beam pipe by using the electrode plates with 
multigrid [24]. The pressure increase has been observed corresponding with the increasing electron current density into the wall. In the RCS, no tune shift relating to the electron cloud hasn't been observed so far. Therefore, the effect of the electron cloud on the dynamic pressure seems to be negligible in the RCS although the conclusive basis should be confirmed by the measurement using the electrode.

From the above considerations, we assume that the leading candidate of the dynamic pressure mechanism in the RCS is the ISD process. The difference in the beam power dependence of the pressure from each situation in Figs. 15 and 16 is mainly due to the difference in the amount of the molecules adsorbed on the wall surface, which is desorbed by the ions accelerated by the beam potential. When the surface density of the adsorbed molecules is large, the pressure largely increases even with a small beam power, because of the large amounts of desorbed molecules per induced ion. More specifically, in point $\mathrm{A}$ of Fig. 14, the pressure runs away up to $10^{-3} \mathrm{~Pa}$, even in the $300 \mathrm{~kW}$ beam operation as shown in Fig. 15(a), because of a large amount of adsorbed molecules during the six-month air exposure in the entire beam lines. The pressure rise in point $\mathrm{C}$ is much smaller than that in point A even with the higher beam power. It is because the amount of adsorbed molecules is kept small due to the continuous evacuation after beam conditioning. The pressure rise in point $\mathrm{D}$ is also much smaller than point $\mathrm{A}$. During the maintenance before point $\mathrm{D}$, only several flanges have been opened to air for a short time in some sections and other sections have been kept in a vacuum with the pumps stopped. Thus the adsorbed molecules on the surface are less than point $\mathrm{A}$. Comparing with point $\mathrm{C}$, the pressure rise in point $\mathrm{D}$ is larger even with similar beam power. A rate of adsorption and a rate of desorption is equal in the equilibrium condition. Henry adsorption isotherm is based on a simple assumption, that the adsorption rate is the impingement rate of the gas molecules to the surface times sticking probability. Under the Henry adsorption isotherm, it is written as

$$
\frac{c P}{\sqrt{2 \pi m k T}}=\frac{q}{\tau},
$$

where $c$ is the sticking probability on the surface, $m$ is the molecule mass, $q$ is the surface density of adsorbed molecules, and $\tau$ is the mean residence time of the molecules on the surface. The term $P / \sqrt{2 \pi m k T}$ represents the molecular impingement rate per unit surface area. Equation (2) represents that in the adsorption equilibrium, the surface density of adsorbed molecules is proportional to the pressure. During the shutdown before point $\mathrm{D}$, the beam line reaches the adsorption equilibrium at about $10^{-2} \mathrm{~Pa}$ in the sections where the vacuum is kept with the pumps stopped (Sec. V B). Thus, a relatively large number of molecules are adsorbed on the surface. Thus, the pressure rise in point $\mathrm{D}$ is larger than that in point $\mathrm{C}$. The relation between the amount of adsorbed molecules during the preceding vacuum system shutdown and the pressure rise is discussed in Sec. V B in more detail.

The molecules on the wall surface are knocked out by the ISD and partially evacuated by the pumps. The beam conditioning effect is obtained by the repetition of such a process, which is characterized by the gradual pressure decrease in the long term. This situation is found in point $\mathrm{B}$ and E in Fig. 14 [Figs. 15(b) and 15(e)]. In a realistic time range, the desorption rate becomes an almost constant value $\Delta Q$ with a unit of $\mathrm{Pa} \mathrm{m}^{3} \mathrm{~s}^{-1}$. When the pumping speed is $S$ with a unit of $\mathrm{m}^{3} \mathrm{~s}^{-1}$, the pressure rise becomes almost constant as $\Delta P=\Delta Q / S$ with a unit of $\mathrm{Pa}$ in this near-equilibrium condition.

As other examples, Figs. 17(a) and 17(b) show the pressure in points $\mathrm{F}$ and $\mathrm{G}$ of Fig. 14, respectively. Each situation is described:

(Point F) The period of the user operation with the $500 \mathrm{~kW}$ beam power. The conditioning effect is observed [Fig. 17(a)].

(Point G) The time of the beam commissioning after the vacuum system shutdown. The beam line has been kept at a vacuum with the pumps stopped and the PV closed during the shutdown except in the third arc section. In the third arc section, the nonevaporable getter (NEG) pumps have been additionally installed for the vacuum improvement during the shutdown period (Sec. VA). The large pressure increase occurs during the $500 \mathrm{~kW}$ beam power operation around the second arc section [Fig. 17(b)].

In the shutdown before point $\mathrm{G}$, the beam line pressure where the vacuum has been kept with the pumps stopped is considered to become around $10^{-2} \mathrm{~Pa}$ (Sec. V B). Relatively large numbers of molecules are adsorbed on the surface in the low-vacuum equilibrium. Thus, the amount of pressure rise in point $\mathrm{G}$ is larger than that at the end of point $\mathrm{F}$ even with the same beam power of $500 \mathrm{~kW}$. The effect of the adsorbed molecules during the preceding vacuum system shutdown on the dynamic pressure is described in Sec. VB. These phenomena are qualitatively consistent with the outgassing mechanism considered above. It is noticed that the degree of the pressure rise is very different in each beam line section in Fig. 17(b). The pressure runaway occurs only in and around the second arc section (C15). The reason for the little pressure rise in the third section might be the vacuum improvement by the additional NEG pumps. However, there is also little pressure rise in the first arc $(\mathrm{C} 02)$ where no additional pump is installed. The beam itself is possible to be another factor related to the pressure rise. If the charge density of the beam in the second arc is larger than that in other sections, the electric field between the beam and the beam pipe is larger. Thus, the energy of the ions bombarding the surface in the second arc section is larger, 

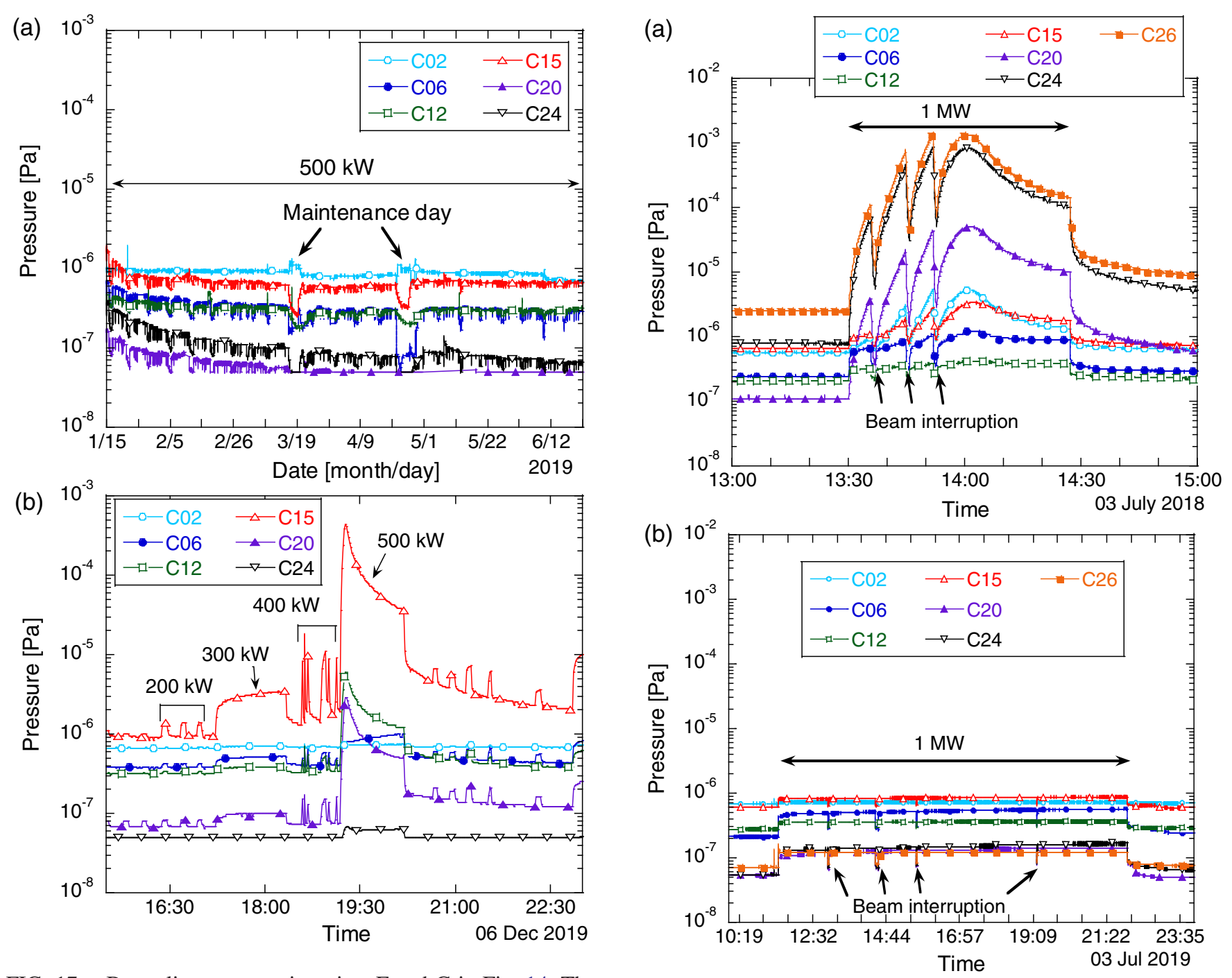

FIG. 17. Beam line pressure in points $F$ and $G$ in Fig. 14. The pressure measured by the same CCGs as Fig. 15 is plotted. (a) [Point F] Pressure during the user operation with the $500 \mathrm{~kW}$ beam power. (b) [Point G] Pressure when high-power beam commissioning is performed after the vacuum system shutdown. The beam line has been kept at a vacuum with the TMPs stopped except in the third arc section, where the NEG pumps are additionally installed in the third arc section during the shutdown (Sec. VA). The pressure value of $5 \times 10^{-8} \mathrm{~Pa}$ is the minimum limit of CCG detection.

and as a result, the desorption rate becomes larger compared to other sections. However, so far, there has been no chance to systematically investigate the relation between the beam condition and pressure rise. That will be a future theme. In this article, the analysis is performed without taking into account such beam conditions.

The pressure in the beam commissioning with $1 \mathrm{MW}$ beam power is shown herein as the final example of the dynamic pressure behavior. So far, the RCS has experienced the rated $1 \mathrm{MW}$ beam power operation tests three

FIG. 18. Dynamic pressure during the $1 \mathrm{MW}$ beam power operation in points $\mathrm{X}$ and $\mathrm{XX}$ in Fig. 14. The pressure measured by the same CCGs as Fig. 15 and the CCG in C26 is plotted. (a) [Point X] Pressure in the first $1 \mathrm{MW}$ beam test. The TMP in C26 has been stopped because of the FRTD trouble. The beam operation is interrupted three times due to the beam loss monitor signal increase caused by the pressure rise. (b) [Point XX] Pressure in the second $1 \mathrm{MW}$ beam test. All TMPs have been uneventfully under operation. The beam operation is interrupted four times due to the trip of the acceleration tube in the linac, etc.

times for $1 \mathrm{~h}$ in $2018,10.5 \mathrm{~h}$ in 2019, and 1.5 days in 2020 . The first and second tests are shown in points $\mathrm{X}$ and $\mathrm{XX}$ in Fig. 14, respectively. The beam line pressure in each operation is shown in panels (a) and (b) of Fig. 18. In the first case shown by panel (a), the TMP in C26 has been stopped because of the FRTD trouble caused by the electrical noise from the pulse magnet. Consequently, the pressure drastically increases by nearly three orders of magnitude in that area. The pressure rise in the second $1 \mathrm{MW}$ beam operation shown in panel (b) is much smaller 
than that in the first case. In both cases, the user operation with the $500 \mathrm{~kW}$ beam power has been carried out until just before these $1 \mathrm{MW}$ beam commissioning. Hence, the conditioning by the continuous desorption of the adsorbed molecules on the wall surface is sufficiently conducted. It can be explained that the pressure runaway, which occurs in the first case, would be caused by the small pumping speed.

\section{B. Analytical calculation}

The analysis of the pressure runaway in the ISR is reported by Gröbner and Calder [16,17]. Their reports show that the gas amount evacuated from the beam line and that released to the gas phase in the beam line are balanced in the equilibrium as follows:

$$
s P=\frac{I}{e} \eta \sigma_{\mathrm{i}} P+Q_{0},
$$

where $Q_{0}$ is the outgassing rate per unit length without the beam, $s$ is the pumping speed per unit length, and $\eta$ is the ISD coefficient, which is the number of gas molecules desorbed per ion. The pressure $P$ becomes

$$
P=\frac{Q_{0}}{s-\frac{I}{e} \sigma_{\mathrm{i}} \eta}
$$

hence, it diverges in the critical beam current $I_{\mathrm{cr}}$ defined as follows:

$$
I_{\mathrm{cr}}=\frac{s e}{\sigma_{\mathrm{i}} \eta} .
$$

The analytical model of the time-dependent behavior of the pressure runaway in the ISR is reported by Kanazawa in 2014 [25]. The time-dependent pressure is given by solving the simultaneous time differential equations for the gas molecule density in the gas phase and the surface density of the adsorbed molecules. The solution well reproduces the time-dependent pressure runaway behavior in the ISR. In this report, Kanazawa's analytical approach is applied to calculate the dynamic pressure behaviors in the RCS.

The time derivative of the gas molecule density and the surface density of the adsorbed molecules during the beam operation is described by the following simultaneous differential equations [25]:

$$
\begin{aligned}
& a \frac{d n}{d t}=\frac{I}{e} \sigma_{\mathrm{i}} \sigma_{d} q\left(n+n_{0}\right)-s n, \\
& h \frac{d q}{d t}=-\frac{I}{e} \sigma_{\mathrm{i}} \sigma_{d} q\left(n+n_{0}\right),
\end{aligned}
$$

where $n$ is the density of the gas phase molecules caused by ion bombardment, $t$ is the time, $n_{0}$ is the density of the gas phase molecules without beam, which means $n(t=0)$, $\sigma_{d}$ is the desorption cross section of the molecules by a
TABLE III. Values of the fixed parameters in the calculation.

\begin{tabular}{lc}
\hline \hline Parameter & Value \\
\hline$\sigma_{i}$ & $1.5 \times 10^{-22} \mathrm{~m}^{2}$ \\
$\sigma_{d}$ & $5.0 \times 10^{-20} \mathrm{~m}^{2}$ \\
$a$ & $0.07 \mathrm{~m}^{2}$ \\
$h$ & $0.9 \mathrm{~m}$ \\
$f$ & $25 \mathrm{~s}^{-1}$ \\
$E$ & $3 \mathrm{GeV}$ \\
$\bar{v}$ & $2.5 \times 10^{8} \mathrm{~m} \mathrm{~s}^{-1}$ \\
$L$ & $348.3 \mathrm{~m}$ \\
\hline \hline
\end{tabular}

bombardment of an ion on the surface, $a$ is the beam pipe cross section, and $h$ is a circumferential length of the beam pipe cross section. There is a relation among $\eta, \sigma_{d}$, and $q$ as $\eta=\sigma_{d} q$. The term $I \sigma_{i}\left(n+n_{0}\right) / e$ is the number of ions, which are the gas molecules ionized by the beam. Table III presents the fixed parameters in the calculation. The typical size of the RCS beam pipe is used for the values of parameters $a$ and $h$, respectively. The ionization cross section $\sigma_{i}$ is calculated by Bethe's formula with the experimentally determined coefficients [26]. The $\sigma_{i}$ value calculated for carbon monoxide is used because it is the main gas component during the highpower operation as shown in Fig. 6(b). The momentum of the proton in the RCS sinusoidally increases during the acceleration time of $20 \mathrm{~ms}$. The corresponding $\sigma_{i}$ during the acceleration time is calculated and the averaged value is used. The same value as that in Kanazawa's report is used for the desorption cross section $\sigma_{d}$ [25]. The corresponding beam current $I$ for a given output beam power is calculated as follows:

$$
I=\frac{P_{\text {beam }}}{f E e} \times e \frac{\bar{v}}{L}=\frac{P_{\text {beam }} \bar{v}}{f E L},
$$

where $P_{\text {beam }}$ is the beam power, $f$ is the repetition rate of the beam, $E$ is the beam energy, $\bar{v}$ is the average beam velocity, and $L$ is the circumferential length of the RCS.

The term $P_{\text {beam }} / f E e$ represents the number of protons in a bunch, while $e \bar{v} / L$ represents the electric charge that passes through a certain cross section per unit time per proton. The pressure is converted by the equation of state $P=\left(n_{0}+n\right) k T$ from the density of the gas molecules $n$ obtained by solving Eq. (6).

In the calculation for comparison with the measurements, the initial surface density of adsorbed molecules $q_{0}$, which is the $q$ value at $t=0$, is adjusted to reproduce the measured pressure behavior. The measured pressures in points $\mathrm{G}$ and $\mathrm{X}$ in Fig. 14 [Figs. 17(b) and 18(a)] are compared with the calculation as typical examples. The $s$ value is calculated by the pumping speed of the TMP and the conductance of the beam pipe to the objective CCG location. The CCGs in C15 and C26, which show the 

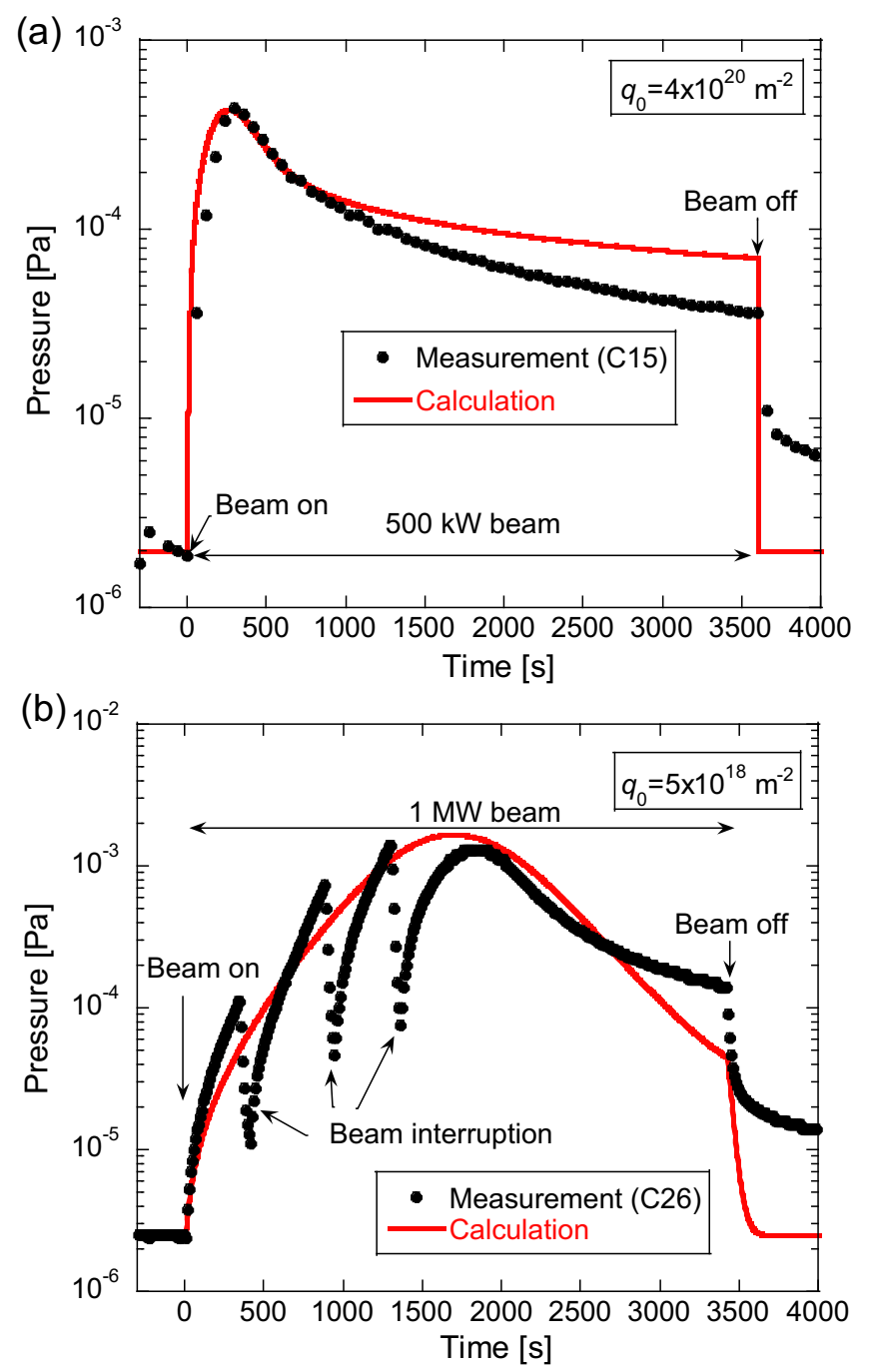

FIG. 19. Comparison of the calculated pressure with the one measured by the CCGs. The origin of the horizontal axis is the time when the beam operation started. The adjusted $q_{0}$ value is also presented in each panel. (a) Pressure in C15 at point G of Fig. 14 during the beam operation with $500 \mathrm{~kW}$ power [Fig. 17(b)]. (b) Pressure in $\mathrm{C} 26$ at point $\mathrm{X}$ of Fig. 14 during the beam operation with $1 \mathrm{MW}$ power [Fig. 18(a)]. The beam interruptions during the beam operation are not considered in the calculation.

largest pressure rise in each case, are selected as representatives, respectively. Each $s$ value is $0.09 \mathrm{~m}^{3} \mathrm{~s}^{-1} \mathrm{~m}^{-1}$ for $\mathrm{C} 15$ in point $\mathrm{G}$ and $0.002 \mathrm{~m}^{3} \mathrm{~s}^{-1} \mathrm{~m}^{-1}$ for $\mathrm{C} 26$ in point $\mathrm{X}$. The pumping speed is very small in the latter because the TMP in C26 is out of operation and the distance to the nearest TMP in operation is $30 \mathrm{~m}$. Figure 19 shows a comparison of the calculated and measured pressures during the beam operation in each case. The beam power is $500 \mathrm{~kW}$ and $1 \mathrm{MW}$, respectively.

The pressure behavior during the beam operation is reproduced by the calculation. There is a disagreement in the time constant of the pressure increase and decrease between the calculation and the measurement. Possible reasons may be the fact that readsorption of the desorbed molecules is not considered, or the $s$ value has some difference with the real pumping speed per unit length, and so on. The adjusted $q_{0}$ value is $4 \times 10^{20} \mathrm{~m}^{-2}$ for point $\mathrm{G}$ and $5 \times 10^{18} \mathrm{~m}^{-2}$ for point $\mathrm{X}$, respectively. These $q_{0}$ values difference are qualitatively understandable. That is, a large number of molecules adsorb on the surface during the system shutdown before point $\mathrm{G}$, while the adsorbed molecules are desorbed and evacuated by the continuous beam conditioning before point $\mathrm{X}$. The $q_{0}$ value of a monomolecular layer is generally considered to be $10^{19} \mathrm{~m}^{-2}$; hence, the adjusted $q_{0}$ value in each case of point $\mathrm{G}$ and $\mathrm{X}$ corresponds to 40 and 0.5 molecular layers, respectively for ideally flat smooth surfaces. Considering the real surface area of the beam pipes, the actual number of molecular layers would become much smaller.

Here, the validity of the $q_{0}$ value is examined in more detail. The mean residence time on the surface $\tau$ in Eq. (2) is written by Frenkel's formula as

$$
\tau=\tau_{0} \exp \left(\frac{E_{\mathrm{des}}}{R T}\right)
$$

where $E_{\text {des }}$ is a desorption energy per mol, $\tau_{0}$ is a constant value that has a similar value to the inverse of the frequency of a molecule on the surface due to thermal vibration [27], and $R$ is the molar gas constant. The generally used $\tau_{0}$ value is $10^{-13} \mathrm{~s}$ [28]. As can be seen from Eq. (8), the mean residence time largely depends on $E_{\text {des. }}$. In a general vacuum system, a typical $E_{\text {des }}$ of the residual gas is thought to be around $70-120 \mathrm{~kJ} / \mathrm{mol}$. Molecules with the smaller $E_{\text {des }}$ than $70 \mathrm{~kJ} / \mathrm{mol}$ are rapidly pumped out due to the very short residence time on the surface, while those with larger $E_{\mathrm{des}}$ than $120 \mathrm{~kJ} / \mathrm{mol}$ continue to be adsorbed due to the long residence time [28]. Carbon monoxide is considered here because it is the main desorbed gas in the beam operation as shown in Fig. 6(b). The total pressure is about $10^{-6} \mathrm{~Pa}$ without beam, and the water is the main residual gas component [Fig. 6(a)]. The partial pressure of carbon monoxide is roughly tens percent of the water. Thus, the partial pressure of carbon monoxide is assumed to be about $10^{-7} \mathrm{~Pa}$ for both points $\mathrm{G}$ and $\mathrm{X}$.

For point $\mathrm{G}$, where the initial surface density $q_{0}$ value is derived to be $4 \times 10^{20} \mathrm{~m}^{-2}$, the corresponding $E_{\text {des }}$ value is $104 \mathrm{~kJ} / \mathrm{mol}$ from Eqs. (2) and (8) with a simple assumption that a sticking probability $c$ is 1 . In the static situation, as mentioned above, the molecules adsorbed with large $E_{\text {des }}$ values are not evacuated due to the long residence time. In the dynamic situation with beam, however, the adsorbed molecules even with large $E_{\text {des }}$ values are desorbed by the ISD because the ions give enough energy to desorb such molecules. Thus, the numbers of adsorption sites with a variety of $E_{\mathrm{des}}$ values become vacant. During the vacuum system shutdown after the beam operation, the molecules are adsorbed on such sites. When the pumping restarts, the 

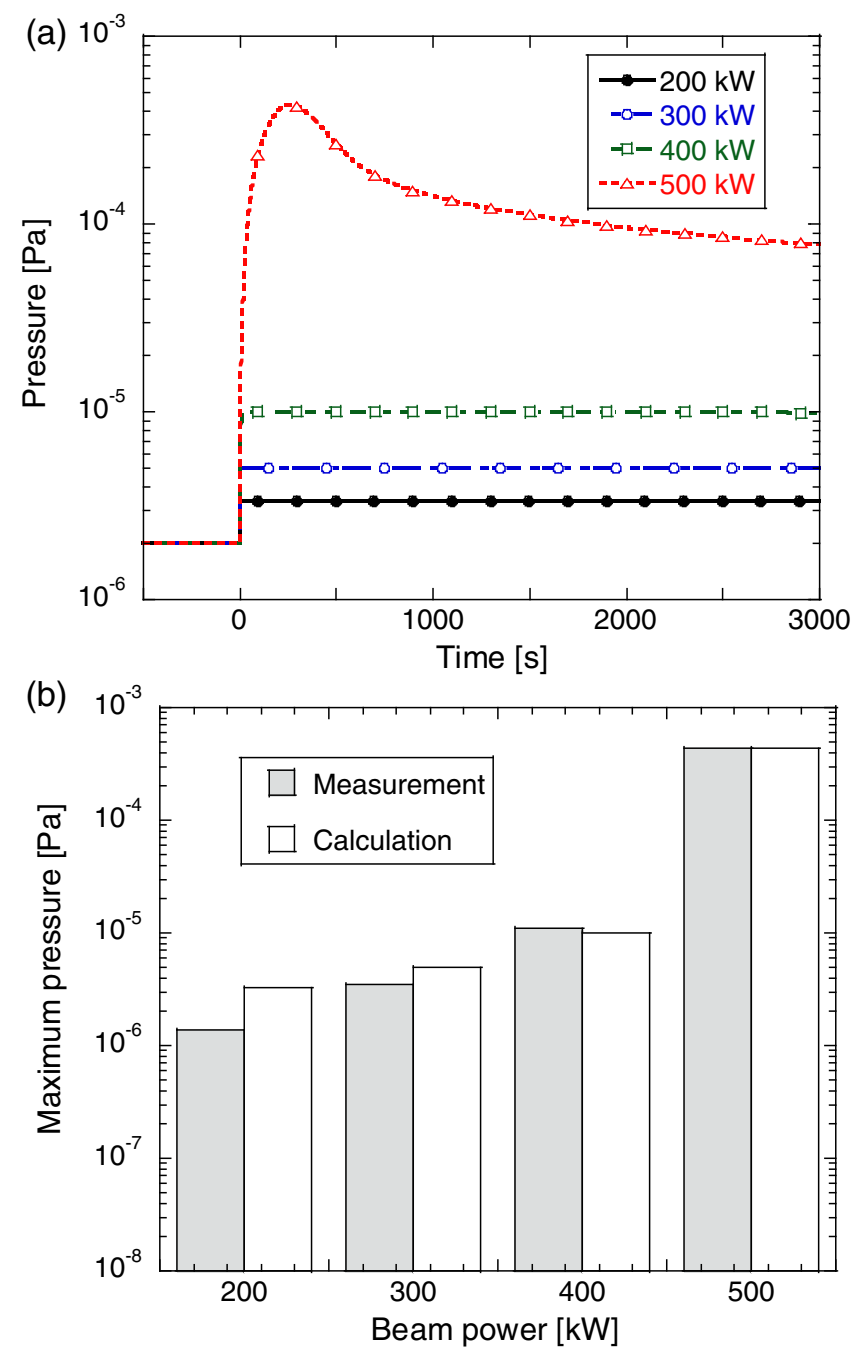

FIG. 20. Dependence of the pressure on the beam power at point G in Fig. 14 [Fig. 17(b)]. In the calculation, the $q_{0}$ value is fixed to $4 \times 10^{20} \mathrm{~m}^{-2}$, which is the value obtained for the case of $500 \mathrm{~kW}$ beam power [Fig. 19(a)]. (a) Calculated time variation of the pressure. The origin of the horizontal axis is set to the time when the beam operation started. (b) Comparison of the calculated maximum pressure with the one measured by the CCG in $\mathrm{C} 15$ at each beam power.

absorbed molecules with small $E_{\text {des }}$ values are evacuated in a short time. However, the molecules adsorbed on the large $E_{\text {des }}$ sites remain on the surface. The estimation result represents that the remained molecules have the $E_{\text {des }}$ value of $104 \mathrm{~kJ} / \mathrm{mol}$ on average in such a situation. Generally, the $E_{\text {des }}$ value of the adsorbed molecules on multilayers is small. For example, Freundlich adsorption isotherm assumes that the $E_{\text {des }}$ decreases exponentially with surface coverage [28]. However, the molecules on the dissociative chemisorption or molecular chemisorption layer would have larger $E_{\mathrm{des}}$ values than physisorption [29]; in general, for the water on a metal surface, the typical $E_{\mathrm{des}}$ value of the dissociative chemisorption is around $120-150 \mathrm{~kJ} / \mathrm{mol}$, that of molecular chemisorption is around $70-120 \mathrm{~kJ} / \mathrm{mol}$, and

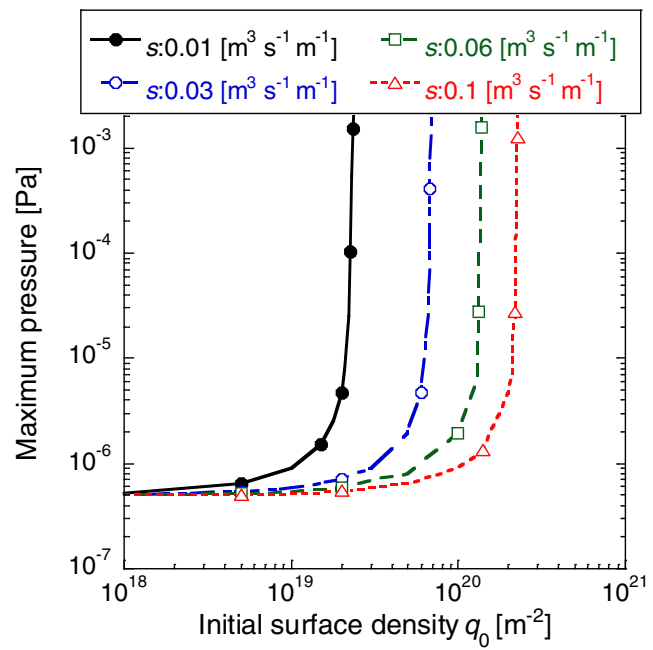

FIG. 21. Calculated dependence of the maximum pressure on the initial surface density of the adsorbed molecules for some pumping speed per unit length values. The beam power is fixed to $1 \mathrm{MW}$. The initial pressure is set to $5 \times 10^{-7} \mathrm{~Pa}$.

that of physisorption is $44-50 \mathrm{~kJ} / \mathrm{mol}$. The surface of the chemisorbed layer is not stable because there are functional groups or atoms, which causes the chemisorption with the molecules of the next layer. Thus, the $E_{\text {des }}$ values of the second and subsequent layers may be much larger than that of physisorption. Thus one of the reasons for the relatively large $E_{\text {des }}$ value averaged for multimolecular layers in point $\mathrm{G}$ may come from these chemisorptions.

For point X, with the $q_{0}$ value of $5 \times 10^{18} \mathrm{~m}^{-2}, E_{\text {des }}$ is estimated to be $93 \mathrm{~kJ} / \mathrm{mol}$ from Eqs. (2) and (8). In the beam conditioning before point $\mathrm{X}$, it is considered that the adsorbed molecules even with large $E_{\text {des }}$ value are desorbed by the ISD and pumped out. Thus, it is considered that the estimated $E_{\text {des }}$ is a reasonable value in such a situation.

A calculation for the cases of the 200,300 , and $400 \mathrm{~kW}$ beam powers in point $\mathrm{G}$ in Fig. 14 is also performed. Only the beam power is changed using the fixed $q_{0}$ value determined by the $500 \mathrm{~kW}$ case; $q_{0}=4 \times 10^{20} \mathrm{~m}^{-2}$. Figure 20(a) shows the calculated time dependence of the pressure for each beam power. The result that the pressure runaway doesn't occur below $500 \mathrm{~kW}$ is consistent with the measurement. The reason that the pressure runaway occurs at the $500 \mathrm{~kW}$ beam power can be explained by the average current $I$ in Eq. (7) getting close to the critical current $I_{\text {cr }}$ in Eq. (5). Panel (b) in Fig. 20 shows a comparison of the maximum pressures between the calculation and the measurement. The calculation well reproduces the measured maximum pressure in each beam power.

The calculation is performed by changing parameters $s$ and $q_{0}$. Figure 21 shows the calculation results of the maximum pressure when the $q_{0}$ value is changed for each $s$ value in the case of $1 \mathrm{MW}$ beam power. In the current system, the average pumping speed per unit length is 


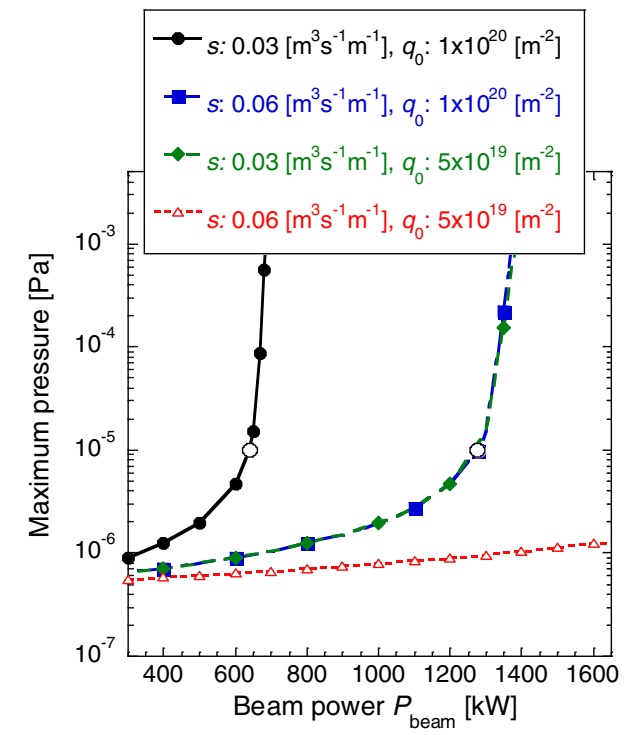

FIG. 22. Calculated dependence of the maximum pressure on the beam power for the combinations of two realistic $s$ and $q_{0}$ values. The initial pressure is set to $5 \times 10^{-7} \mathrm{~Pa}$. The open circles represent the critical beam power $P_{\text {beam_cr }}$ in each combination of $s$ and $q_{0}$ values. The $P_{\text {beam_cr }}$ for the combination of $s$ : $0.06 \mathrm{~m}^{3} \mathrm{~s}^{-1} \mathrm{~m}^{-1}$ and $q_{0}: 5 \times 10^{19} \mathrm{~m}^{-2}$ is out of range, which is $2.6 \mathrm{MW}$.

approximately $0.03-0.04 \mathrm{~m}^{3} \mathrm{~s}^{-1} \mathrm{~m}^{-1}$ because the TMPs with $1.3 \mathrm{~m}^{3} \mathrm{~s}^{-1}$ pumping speed for nitrogen are installed at a 30-40 m interval. In each $s$ case, the pressure runaway occurs as the $q_{0}$ value increases. This is because the critical beam current decreases and is getting close to the beam current when the $q_{0}$ value increases. When the $s$ value is larger, the $q_{0}$ value, with which the pressure runaway occurs, becomes larger. Figure 22 shows the calculation results of the maximum pressure dependence on the beam power for some combinations of $q_{0}$ and $s$ values. The realistic improvement of the pumping speed per unit length is to reduce the interval distance between the pumps to about half, corresponding to make $s$ the double. Thus, the calculation results with the $s$ values of 0.03 and $0.06 \mathrm{~m}^{3} \mathrm{~s}^{-1} \mathrm{~m}^{-1}$ are examined. The pressure doesn't diverge even in the higher beam power with the smaller $q_{0}$ and the larger $s$. This is because the smaller $q_{0}$ and the larger $s$ give the larger critical beam current. The open circles in Fig. 22 represent the critical beam power $P_{\text {beam_cr }}$ for each combination of $s$ and $q_{0}$ obtained by Eqs. (5) and (7). In conclusion, the increase of the pumping speed and the reduction of the adsorbed molecules on the surface are effective approaches for preventing the pressure runaway in the high-power beam operation.

\section{COUNTERMEASURE AGAINST PRESSURE RUNAWAY}

The pressure runaway in the ISR of CERN has been finally suppressed by adding the vacuum pumps, such as titanium sublimation pumps, to increase the pumping speed per unit length $s[16,17]$. They also have performed offline discharge cleaning and in situ baking around $300{ }^{\circ} \mathrm{C}$ to reduce the initial ISD coefficient $\eta_{0}$. Reducing $\eta_{0}$ means reducing $q_{0}$ from the relation $\eta_{0}=\sigma_{\mathrm{d}} q_{0}$. Thus, our conclusion for suppressing the pressure runaway is the same as the ISR point of view. In the RCS, $s$ can be increased by adding new pumps in the ports that are not currently used. However, applying offline discharge cleaning and in situ baking is difficult because of several reasons. As mentioned in Sec. I, alumina ceramics beam pipes are installed in the magnets to prevent eddy currents. A TiN film with a thickness of approximately $10 \mathrm{~nm}$ is coated on the surface of the alumina ceramics to suppress the secondary electron emission. Discharge cleaning is a method of desorbing the surface molecules by hitting ions such as $\mathrm{Ar}^{+}$with an energy of a few $\mathrm{keV}$ on the duct surface; thus, the coating will be in danger of being sputtered away. Besides, the thermal expansion during baking performed around $300^{\circ} \mathrm{C}$ can break the alumina ceramics because of an unacceptably large inner stress from the nearby metal parts. The $q_{0}$ value must be suppressed without such risks. In an adsorption equilibrium in Eq. (2), the surface density of the adsorbed molecules is smaller when the equilibrium pressure is lower. Thus, it is effective for the $q_{0}$ suppression to make the base pressure as low as possible. Thus, the additional installation of the pumps might have advantages not only for the $s$ increase but also for the $q_{0}$ suppression. The main gas components in the beam line are water and hydrogen without beam and carbon monoxide with beam, respectively, as shown in Fig. 6. We consider that the addition of the NEG pump in the beam line is effective because it has a high-pumping speed for such gas species. The NEG pump is composed of several metals with the function of adsorbing or absorbing the gas molecules on the surface or in the bulk [30]. Once the NEG pump is activated by raising the temperature to a certain level, it obtains a getter function even after cooling to room temperature until the surface or the bulk is saturated with the adsorbed or absorbed molecules. Thus, it could suppress the adsorbed molecules on the surface during the vacuum system shutdown without a purge.

\section{A. Pumping speed improvement}

The NEG pumps have been additionally installed in the third arc section of the RCS to investigate their effectiveness. Figure 23 shows the vacuum system diagram of the third arc section. The NEG pumps are installed in the position of the ion pumps that had not been operated. The CapaciTorr ${ }^{\circledR}$ D3500 of the SAES group [31] is selected as the NEG pump with the largest pumping speed, which can be inserted in the port. The effect of the NEG pumps is evaluated using the Molflow+ code, which is a Monte Carlo simulation code in the molecular flow region [32]. The code has many proven records in an accelerator and other 
3rd arc section

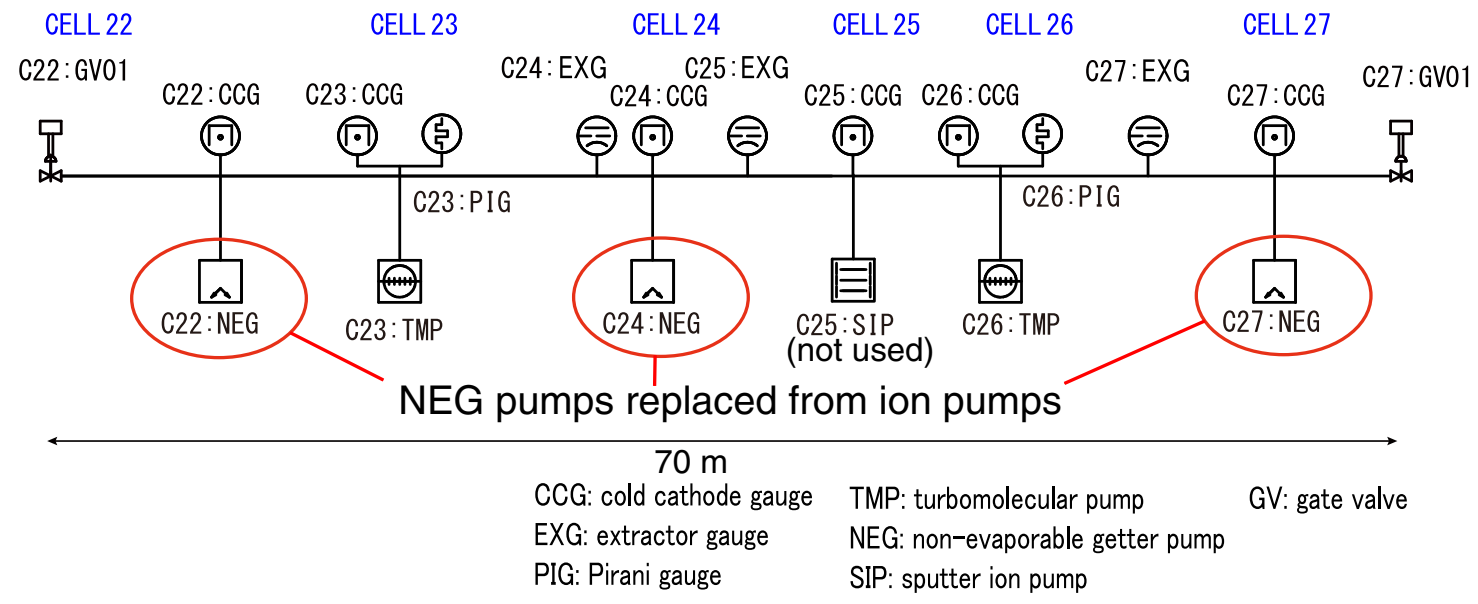

FIG. 23. Layout of the vacuum system for the third arc section, where the NEG pumps are additionally installed. Three NEG pumps are installed in the position of the ion pumps.

vacuum fields [33,34]. Figure 24 shows the model of the third arc section for the simulation. The pumping speed of $1.3 \mathrm{~m}^{3} \mathrm{~s}^{-1}$ and $1.0 \mathrm{~m}^{3} \mathrm{~s}^{-1}$ is used for the TMP and the NEG pump, respectively. The typical outgassing rate per unit area for the unbaked metal such as stainless steel or titanium after weeks of evacuation is generally around $10^{-9} \mathrm{~Pa} \mathrm{~m}^{3} \mathrm{~s}^{-1} \mathrm{~m}^{-2}[6,35]$. Thus the outgassing rate per unit area of $1 \times 10^{-9} \mathrm{~Pa} \mathrm{~m}^{3} \mathrm{~s}^{-1} \mathrm{~m}^{-2}$ is used for the input value for the beampipe wall. The gas flow rates from the upstream and the downstream of the third arc section are the input as a boundary condition. The gas flow from upstream and downstream is decided such that the calculated pressure at the gauge position agrees with the measured pressure in the case without NEG pumps. Figure 25 shows the pressure distribution of the third arc section. The horizontal axis is the distance, whose origin is the uppermost stream part of the third arc section. The dashed and solid lines denote the calculated pressure without and with the NEG pump, respectively. The open triangles and circles represent the pressure measured by the CCGs and extractor gauges (EXGs) before the NEG pump installation. The closed triangles and circles represent those after the NEG pump installation. All values are total pressures for nitrogen equivalent. The pressure value of $5 \times 10^{-8} \mathrm{~Pa}$ is the minimum limit of CCG detection; thus, the calculation cannot be compared with the measurement in those points. The pressure distribution by the simulation is reliable because the calculation and the measurement agree well at the points, where the pressure is in the detectable range of the gauges. Consequently, it is confirmed that the pressure decreases at every point along the beam line by adding the NEG pumps, indicating that the larger pumping speed per unit length $s$ is obtained everywhere in the beam line. The NEG pump is certainly effective for pressure runaway suppression because the large pumping speed and the low-equilibrium pressure are achieved.

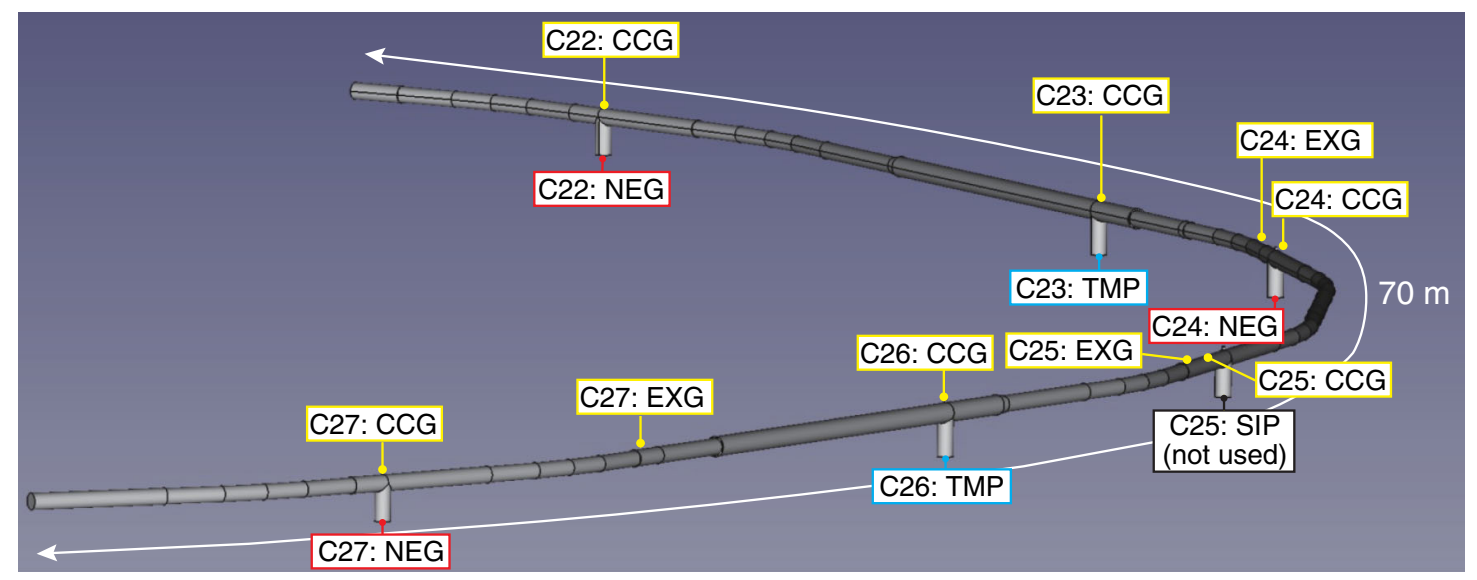

FIG. 24. Model of the Molflow+ calculation for the third arc section. The positions of the gauges and the pumps are also depicted. 


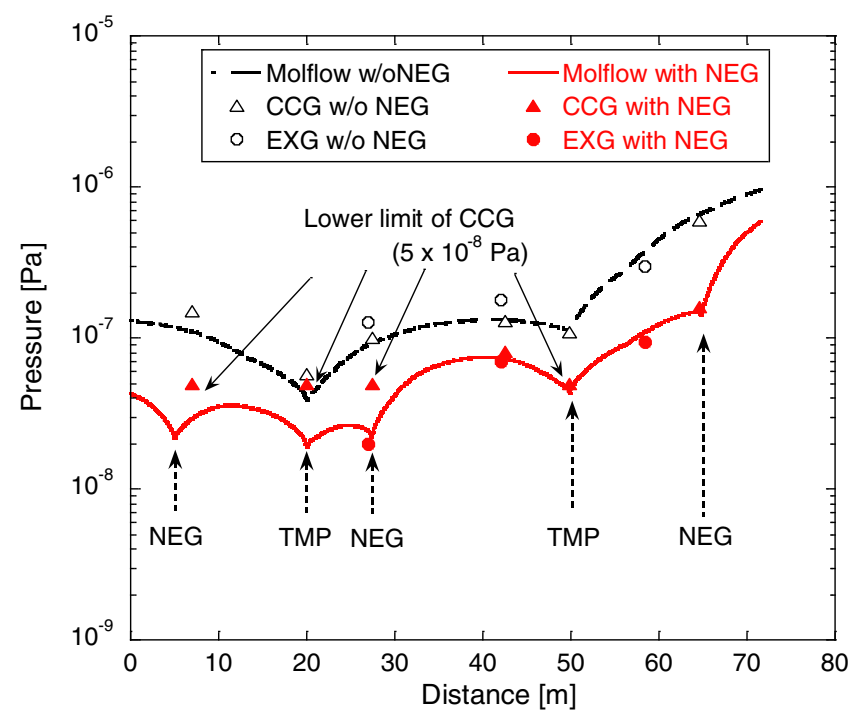

FIG. 25. Pressure distribution in the third arc section before and after the NEG pump installation. The dashed and solid lines denote the calculated pressure by Molflow+ without and with NEG pumps, respectively. The open triangles and circles represent the pressure without the NEG pumps measured by the CCGs and the EXGs, respectively. The closed triangles and circles represent those with NEG pumps. The pressure value of $5 \times 10^{-8} \mathrm{~Pa}$ is the minimum limit of CCG detection.

\section{B. Adsorbed molecules reduction during the vacuum system shutdown}

The effect of the NEG pump on the suppression of the surface density of the adsorbed molecules is investigated by performing a buildup test of the beam line. The pressure behaviors of the second arc section, in which the NEG pump is not installed, and the third arc section with the NEG pumps installed are compared. The NEG pumps in the third arc section are already activated. The equipment layout of each arc section is the same, except for the NEG pump installation. The beam line gate valves are closed to separate each section, all TMPs in the second and third sections are stopped and the pneumatic valves; PVs in Figs. 3 and 11 are closed for the buildup of each section. Figures 26 and 27 show the buildup test results for the total pressure and the ion current of RGA, respectively. After $40 \mathrm{~h}$ from the start of the buildup, the pressure in the second arc section reaches $10^{-3} \mathrm{~Pa}$, while that in the third arc section maintains the low value of $10^{-5} \mathrm{~Pa}$ order. After $250 \mathrm{~h}$, the pressure in the second arc section approaches $10^{-2} \mathrm{~Pa}$, while that in the third arc section is still around $10^{-4} \mathrm{~Pa}$. The main gas component during the buildup is very different from each section (Fig. 27). Hydrogen is the most common gas species in the second arc section without NEG pumps. On the other hand, argon is much larger than other gases in the third arc section with NEG pumps because the NEG pump doesn't adsorb the rare gases. The source of the argon is not an air leak because the leak late

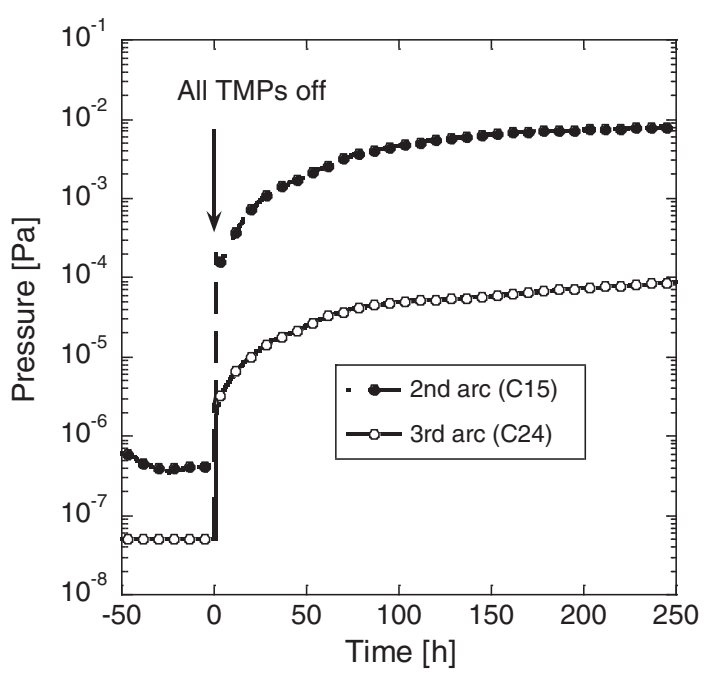

FIG. 26. Result of the buildup test. Time variation of the pressure measured by the CCGs in the second arc section without NEG pumps and the third arc section with NEG pumps. The origin of the horizontal axis is set to the start of the buildup test.

less than $10^{-11} \mathrm{~Pa} \mathrm{~m}^{3} \mathrm{~s}^{-1}$ has been checked for all the beam line components and the connection points of flanges. The argon gas for purging the beam line may be the source of this phenomenon. We plan to examine the effect of the purge gas on the NEG pump and titanium chamber by the offline experiment.

The ion current of gases such as water, carbon monoxide, oxygen, and carbon dioxide decrease after 10-40 h, while the ion current of hydrogen continues to increase to an equilibrium state as shown in Fig. 27(a). Water, carbon monoxide, oxygen, and carbon dioxide are the gas species, which easily stick to the titanium surface. One possibility of this phenomenon is the following. The molecules in the gas phase increase at the start of the buildup, which corresponds to a decrease of the adsorbed molecules on the surface from the conservation of the molecule number. The decrease of the adsorbed molecules makes the increase of the vacant adsorbent sites. Then, the impingent molecules from the gas phase to the surface are adsorbed on such sites. That corresponds to the decrease of the gas phase molecules and the increase of the molecules on the surface. The pressure decreases in such a phase. The system will go to an equilibrium state on a certain isotherm after repeating such a situation. In contrast to those gases, hydrogen diffuses from the bulk of the beampipe and desorbs to the gas phase. That is, the sum of the number of the molecules in the gas phase and on the surface is not conserved because there is a supply source of hydrogen. Thus the hydrogen in the gas phase continues to increase to a certain equilibrium state.

Here, we discuss the amount of the molecules adsorbed during the vacuum system shutdown during which the beam line is kept in a vacuum with the TMPs stopped. 

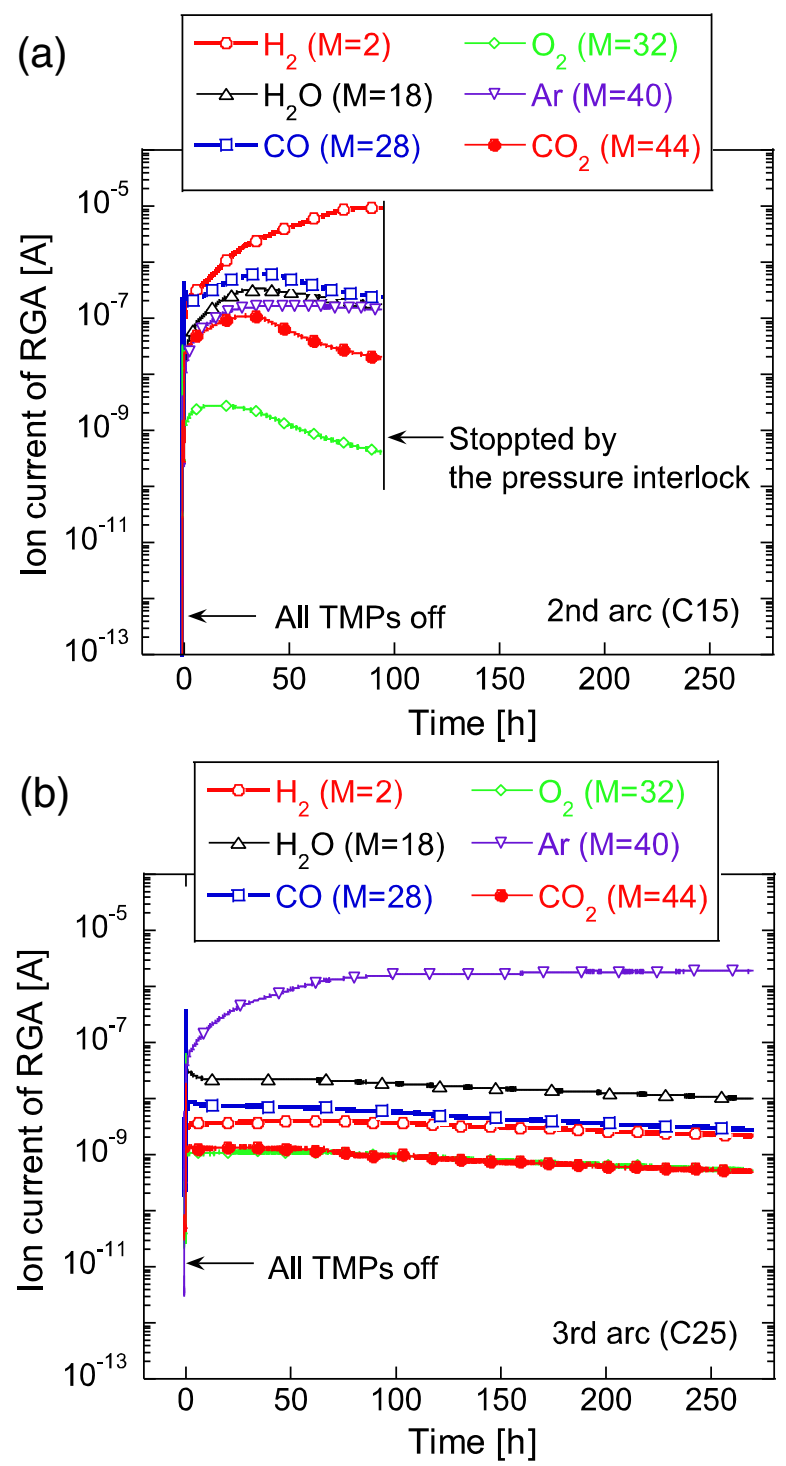

FIG. 27. Time variation of the RGA ion current for typical gas mass numbers during the buildup test. The origin of the horizontal axis is set to the time when the TMPs are stopped. (a) Result of the second arc section without NEG pumps. (b) Result of the third arc section with NEG pumps. In the second arc, the RGA is switched off at around $94 \mathrm{~h}$ by the interlock which is set for the total pressure to $2 \times 10^{-3} \mathrm{~Pa}$. It should be noted that the absolute ion current between the different RGAs cannot be compared because their sensitivities are different.

Carbon monoxide, which is the main desorbed gas during the beam operation (Fig. 6), is examined. In the second arc section without NEG, the main component during the buildup is hydrogen and the total pressure is about $10^{-2} \mathrm{~Pa}$. Because the RGA ion current of carbon monoxide is about one-hundredth of hydrogen, the partial pressure of the carbon monoxide is roughly $10^{-4} \mathrm{~Pa}$ during the system shutdown. On the other hand, in the third arc section, the main component during the buildup is argon and the total pressure is about $10^{-4} \mathrm{~Pa}$. Carbon monoxide is about one-thousandth of argon. The corresponding partial pressure of carbon monoxide is roughly $10^{-7} \mathrm{~Pa}$. Thus, the NEG pump can suppress the adsorbed amount of carbon monoxide by about three orders of magnitude less than that without the NEG pump during the vacuum system shutdown with the TMPs stopped. From the measured results as shown in Fig. 15, it is also obvious that the pressure rise in the beam operation has been more suppressed when the lower beam line pressure is maintained during the precedent maintenance period. From these facts, it is considered that maintaining pressure as low as possible during the vacuum system shutdown by the additional NEG pumps would contribute to reducing the $q_{0}$ and suppression of the pressure runaway.

The NEG pump sorption capacity, which means the maximum amount of absorbed or adsorbed molecules, is $540 \mathrm{~Pa} \mathrm{~m}^{3}$ for hydrogen and $1.6 \mathrm{~Pa} \mathrm{~m}^{3}$ for carbon monoxide according to the CapaciTorr ${ }^{\circledR}$ D3500 manual. The sorption capacity of hydrogen is much higher than that of carbon monoxide because hydrogen diffuses into NEG material, while carbon monoxide is chemically adsorbed on the NEG surface [30]. From the sorption capacity and partial pressure results during the buildup, the NEG pump will be saturated for 170 days by hydrogen and 100 days by carbon monoxide. Usually, the RCS maintenance period with vacuum system shutdown is about three months. The TMPs should be restarted as promptly as possible when the vacuum system becomes ready to operate to avoid surface saturation by carbon monoxide.

\section{Pressure runaway suppression}

The NEG pumps have been installed in the third arc section during the vacuum system shutdown period in 2019. The dynamic pressure after the maintenance period is already shown in Figure 17(b). Regrettably, it has been difficult to measure the effectiveness of the NEG pumps on the pressure rise during the high-power beam operation because of the beam condition change before and after the NEG installation, as described in Sec. IV A. However, because the effect of the additional NEG pumps has been confirmed in the third arc section, the NEG pumps have also been installed in the second arc section in the maintenance period of 2020 with the same configuration as the third arc section (Fig. 23). And fortunately, we have had an opportunity to measure the pressure with the same beam condition as point G in Fig. 14 [Fig. 17(b)]. Figure 28 shows the dynamic pressure after the NEG pump installation in the second arc section. Figure 29 shows the beam power dependence of the pressure without and with NEG pumps in the second arc section. The pressure rise is suppressed by one order of magnitude by the additional NEG pumps. 


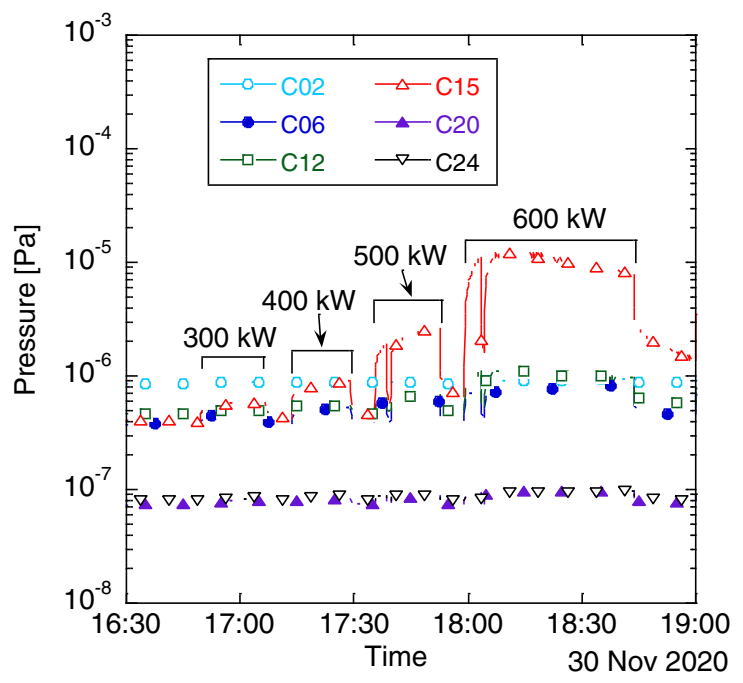

FIG. 28. Pressure measured by the CCGs during the beam operation after the installation of the additional NEG pumps in the second arc section. Other conditions are the same as point $\mathrm{G}$ in Fig. 14 [Fig. 17(b)].

\section{Operation and maintenance}

The NEG pump must be reactivated by increasing the temperature to obtain a fresh surface when the NEG materials are saturated by gas adsorption and absorption. The NEG materials saturate by opening the beam line to air or long-term usage in the vacuum. When the vacuum system operates in normal condition, the activation once per year is enough. In the activation process, the released gas from the NEG pump must be evacuated by other pumps, such as the TMP. The TMP is the main pump in the

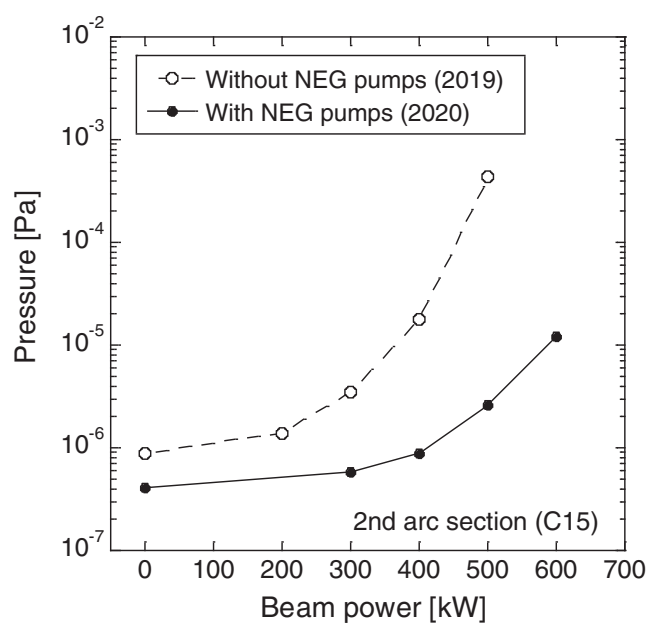

FIG. 29. Beam power dependence of the pressure measured by the CCG in C15 of the second arc section without and with NEG pumps during the beam operation. Each data is obtained in 2019 and 2020, respectively. The maximum pressure in each beam power operation is plotted. The values are obtained from Figs. 17(b) and 28 for the case without and with NEG pumps, respectively.

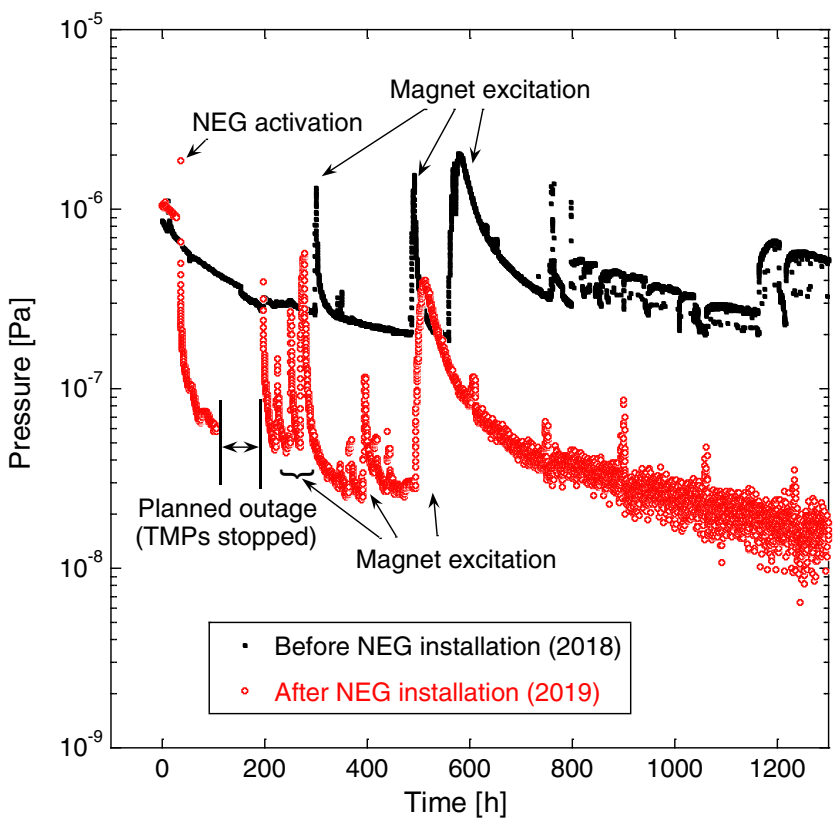

FIG. 30. Pumping curve measured by the EXG in C24 of the third arc section before and after NEG installation. The origin of the horizontal axis is the time after one day of pumping by the TMPs.

RCS vacuum system; hence, a portable TMP pumping unit does not need to be installed during each reactivation work. Thus, the vacuum system, whose main pump is a combination of the TMP and the NEG pump, is suitable from the operating and maintenance points of view. Figure 30 shows the pumping curve in the third arc section before and after the installation of NEG pumps. Each data is obtained in the year 2018 and 2019, respectively. The pressure reaches about $1 \times 10^{-6} \mathrm{~Pa}$ after about one day of evacuation by the TMPs in both cases. In the example of 2019, the pressure of the order of $10^{-8} \mathrm{~Pa}$ is immediately obtained by activating the NEG pump. There is a planned electrical outage for three days in 2019. It is also shown that the pressure of the order of $10^{-8} \mathrm{~Pa}$ is easily recovered from the vacuum system shutdown due to the planned outage.

It is confirmed that both the increase of $s$ and the suppression of the $q_{0}$ is effectively achieved by the additional installation of NEG pumps. The NEG pumps are planned to be installed in all the beam line sections in future work.

\section{SUMMARY}

The events beyond the design assumptions of the vacuum system have occurred in the high-power beam operation in the RCS. The failure of the TMP system and the beam line pressure runaway are typical examples.

The new TMP controller has been developed to connect more than $200 \mathrm{~m}$ cables for installing in an area without radiation influence. The TMP with FRTD tolerability has been newly developed by applying an improved bearing. 
The TMP system becomes more tolerant to a large loss of the high-intensity beam by using such controller and TMP.

The pressure rise during the high-power operation has been systematically investigated. It is found that the pressure nonlinearly increases with increasing the beam power, and the conditioning effect exists. These phenomena are explained by the outgassing mechanism based on the ISD. The validity of this outgassing mechanism is supported by reproducing a measurement with analytical calculation. The calculation also shows that the pressure runaway can suppress when the pumping speed per unit length $s$ is large and the initial surface density of the adsorbed molecules $q_{0}$ is small.

NEG pump has been selected to suppress the pressure runaway. The effectiveness of the additional NEG pumps on the increase of $s$ and the suppression of the $q_{0}$ is examined. The $s$ increase in the entire beam line is confirmed by the simulation and the measurement. The low pressure obtained by the large $s$ also contributes to the small $q_{0}$ in the adsorption equilibrium. The ability of the NEG pump to maintain low pressure during the vacuum system shutdown has been confirmed by the buildup test. The measured dynamic pressure before and after NEG pumps installation with the same beam condition shows that the NEG pumps are effective to suppress the pressure runaway. The vacuum system with the combined TMPs and NEG pumps can easily achieve a pressure of a $10^{-8} \mathrm{~Pa}$ order. We plan to apply the obtained results to the entire RCS vacuum system in future work.

\section{ACKNOWLEDGMENTS}

The authors thank Mr. T. Mabuchi and Mr. M. Sakurai of Osaka Vacuum, Ltd. for their support to provide the improved TMP controller and the TMP with improved bearing. The authors also thank Dr. Hatakeyama for providing the information on the beam monitor. The authors also thank Dr. Saha and Dr. Shobuda for the helpful comments about the beam condition and the electron cloud instability. The authors are also grateful to Dr. M. Kinsho for his encouragement for pushing forward with this work. The authors also give thanks to all the RCS members for their continuous support and daily useful discussions.

[1] H. Hotchi et al., Beam commissioning of the 3-GeV rapid cycling synchrotron of the Japan Proton Accelerator Research Complex, Phys. Rev. ST Accel. Beams 12, 040402 (2009).

[2] High-intensity Proton Accelerator Project Team, Accelerator design report for high intensity proton accelerator facility project, J-PARC, Report No. JAERI-Tech 2003044, Japan Atomic Energy Research Institute, Tokai, Naka, Ibaraki, Japan, 2003.

[3] H. Hotchi, H. Harada, N. Hayashi, S. Kato, M. Kinsho, K. Okabe, P. K. Saha, Y. Shobuda, F. Tamura, N. Tani,
Y. Watanabe, K. Yamamoto, M. Yamamoto, Y. Yamazaki, and M. Yoshimoto, Achievement of a low-loss 1-MW beam operation in the $3-\mathrm{GeV}$ rapid cycling synchrotron of the Japan Proton Accelerator Research Complex, Phys. Rev. Accel. Beams 20, 060402 (2017).

[4] J. Kamiya, Vacuum technologies in high power proton accelerators, J. Vac. Soc. Jpn. 59, 213 (2016).

[5] N. Ogiwara, Ultrahigh vacuum for high intensity proton accelerators: Exemplified by the $3 \mathrm{GeV}$ RCS in the J-PARC, in Proceedings of the International Particle Accelerator Conference 2011, San Sebastián, Spain, p. 971.

[6] J. Kamiya, Y. Hikichi, M. Kinsho, and N. Ogiwara, Titanium alloy as a potential low radioactivation vacuum material, J. Vac. Sci. Technol. A 33, 031605 (2015).

[7] M. Kinsho, Y. Saito, Z. Kabeya, and N. Ogiwara, Titanium flanged alumina ceramics vacuum duct with low impedance, Vacuum 81, 808 (2007).

[8] J. Kamiya, Y. Hikichi, Y. Namekawa, K. Takeishi, T. Yanagibashi, M. Kinsho, K. Yamamoto, and A. Sato, Improvements of vacuum system in J-PARC $3 \mathrm{GeV}$ synchrotron, J. Phys.: Conf. Ser. 874, 012099 (2017).

[9] M. Kinsho, N. Ogiwara, K. Wada, M. Yoshida, T. Nakayasu, and Y. Yamato, Gamma-ray irradiation experiment of turbo molecular pump, Vacuum 73, 175 (2004).

[10] K. Wada, T. Inohara, M. Yoshida, Y. Yamato, T. Nakayasu, M. Iguchi, Y. Hikichi, N. Ogiwara, and K. Mio, Development of the radiation-hardened magnetically suspended compound molecular pump, Vacuum 84, 699 (2009).

[11] N. Ogiwara, M. Kinsho, J. Kamiya, K. Yamamoto, M. Yoshimoto, Y. Hikichi, K. Kanazawa, K. Mio, Y. Takiyama, K. Suganuma, and Y. Saito, Vacuum system for the 3-GeV RCS in J-PARC, Vacuum 84, 723 (2009).

[12] J.F. O'Hanlon, A User's Guide to Vacuum Technology, 3rd ed. (John Wiley \& Sons, Inc., Hoboken, NJ, 2003), p. 209.

[13] H. Hotchi, H. Harada, N. Hayashi, M. Kinsho, K. Okabe, P. K. Saha, Y. Shobuda, F. Taumra, K. Yamamoto, M. Yamamoto, and M. Yoshimoto, J-PARC 3-GeV RCS: 1-MW beam operation and beyond, J. Instrum. 15, P07022 (2020).

[14] O. B. Malyshev, Vacuum in Particle Accelerators: Modelling, Design and Operation of Beam Vacuum Systems, (Wiley-VCH Verlag GmbH \& Co. KGaA, Weinheim, 2020), pp. 421-422.

[15] E. Fischer, Two kilometers at $10^{-10}$ Torr. The CERN intersecting storage rings for protons, J. Vac. Sci. Technol. 9, 1203 (1972).

[16] O. Gröbner and R. S. Calder, Beam induced gas desorption in the CERN intersecting storage rings, in Proceedings of the fifth IEEE Particle Accelerator Conference, San Francisco, CA, USA (1973), p. 760.

[17] R. S. Calder, Ion induced gas desorption problems in the ISR, Vacuum 24, 437 (1974).

[18] https://www.osakavacuum.co.jp/en/.

[19] https://www.hitachi-metals.co.jp/e/product/finemet/cmc/ core/.

[20] K. Ohmi, T. Toyama, and C. Ohmori, Electron cloud instability in high intensity proton rings, Phys. Rev. ST Accel. Beams 5, 114402 (2002).

[21] T. Toyama and the e-cloud study team, Electron cloud effects in the J-PARC rings and related topics, in Proceedings 
of the 31st Advanced ICFA Beam Dynamics Workshop on Electron-Cloud Effects, Tsukuba, Japan (2005), p. 77.

[22] V. Baglin, J. Bojko, O. Gröbner, B. Henrist, N. Hilleret, C. Scheuerlein, and M. Taborelli, The secondary electron yield of technical materials and its variation with surface treatments, in Proceedings of the seventh European Particle Accelerator Conference, Vienna, Austria (2000), p. 217.

[23] W. Fischer, J. M. Brennan, M. Blaskiewicz, and T. Satogata, Electron cloud measurements and simulations for the Brookhaven relativistic heavy ion collider, Phys. Rev. ST Accel. Beams 5, 124401 (2002).

[24] W. Fischer, M. Blaskiewicz, J. M. Brennan, H. Huang, H.-C. Hseuh, V. Ptitsyn, T. Roser, P. Thieberger, D. Trbojevic, J. Wei, S. Y. Zhang, and U. Iriso, Electron cloud observations and cures in the relativistic heavy ion collider, Phys. Rev. ST Accel. Beams 11, 041002 (2008).

[25] K. Kanazawa, On the understanding of the pressure bump, Vacuum 101, 142 (2014).

[26] CAS_CERN Accelerator School: Vacuum Technology, edited by S. Turner, (CERN, Geneva, 1999) p. 169.

[27] J. H. de Boer and F. A. Baker, Bond strength and mean time of adsorption, Vacuum 16, 309 (1966).
[28] K. Jousten, Handbook of Vacuum Technology, 2nd ed. (Wiley-VCH Verlag GmbH \& Co. KGaA, Weinheim, 2016), pp. 238-246.

[29] D. Costa, K. Sharkas, M. M. Islam, and P. Marcus, Ab initio study of the chemical states of water on $\mathrm{Cr}_{2} \mathrm{O}_{3}(0001)$ : From the isolated molecule to saturation coverage, Surf. Sci. 603, 2484 (2009).

[30] S. Borichevsky, Understanding Modern Vacuum Technology, 2nd ed. (Blue Dasher, Ipswich, Massachusetts, 2017), pp. 291-307.

[31] https://www.saesgetters.com.

[32] R. Kersevan and J.-L. Pons, Introduction to Molflow+: New graphical processing unit-based Monte Carlo code for simulating molecular flows and for calculating angular coefficients in the compute unified device architecture environment, J. Vac. Sci. Technol. A 27, 1017 (2009).

[33] K. Shiabata, Y. Suetsugu, T. Ishibashi, M. Shirai, S. Terui, K. Kanazawa, and H. Hisamatsu, Commissioning of vacuum system for SuperKEKB positron damping ring, J. Vac. Sci. Technol. A 37, 041601 (2019).

[34] F. Chill, S. Wilfert, and L. Bozyk, Cryopumping of hydrogen on stainless steel in the temperature range between 7 and 18 K, J. Vac. Sci. Technol. A 37, 031601 (2019).

[35] R. J. Elsey, Outgassing of vacuum materials-I, Vacuum 25, 299 (1975). 